\title{
A Generalization of the Classical Moment Problem on *-Algebras with Applications to Relativistic Quantum Theory. II.
}

\author{
Michel Dubois-Violette \\ Laboratoire de Physique Théorique et Hautes Énergies, Université de Paris XI, F-91405 Orsay, France*
}

\begin{abstract}
We discuss some properties of a non-commutative generalization of the classical moment problem (the $m$-problem) previously introduced. It is shown that there is a connexion between the determination of the problem and the self-adjointness properties in the corresponding Hilbert space. This generalizes the well-known connexion between the determination of the measure in the classical moment problem and the self-adjointness properties of the polynomials as operators in the corresponding $L^{2}$-space. The dependence of the $m$-problem on the choice of $C^{*}$-semi-norms and on the action of $*_{\text {- }}$ homomorphisms is also investigated. As an application, it is shown that if a quantum field (in a very general sense) is essentially self-adjoint then the $m$ problem for the Wightman functional is determined on the quasi-localizable $C^{*}$-algebra and that the corresponding representation of the localizable algebra generates the bounded observables of the field. It is pointed out that (ultraviolet and spatially) cut-off fields fall in this class and, therefore, are in one to one correspondance with states on the quasi-localizable $C^{*}$-algebra.
\end{abstract}

\section{Introduction}

This paper is a continuation of a preceding one [1] hereafter referred as Part I. Its object is to complete the algebraic discussion of the non-commutative generalization of the classical moment problem (the $m$-problem) introduced in Part I and to extend the applications to quantum field theory.

Let us first describe an important result on the classical moment problem [3-5] which will be generalized in this paper. Let $\phi$ be a positive linear form on the ${ }^{*}$ algebra $\mathbb{C}[X]$ of the complex polynomials with respect to one indeterminate $X$. Basically, to solve the moment problem for $\phi$ means to produce a self-adjoint operator $\pi(X)$ in a Hilbert space $\mathfrak{S}$ with a vector $\Omega \in \bigcap_{n \geqq 0} \operatorname{dom}\left(\pi(X)^{n}\right)$ in such a way

* Laboratoire associé au Centre National de la Recherche Scientifique. Postal address : Laboratoire de Physique Théorique et Hautes Énergies, Bâtiment 211, Université de Paris-Sud, F-91405 Orsay, France 
that $\Omega$ is cyclic for the von Neumann algebra $\{\pi(X)\}^{\prime \prime}$ and that the equalities $\phi\left(X^{n}\right)$ $=\left(\Omega \mid \pi(X)^{n} \Omega\right)$ hold for all integers $n \geqq 0$. Indeed the corresponding conventional solution is the positive rapidly decreasing measure $\mu$ on $\mathbb{R}$ such that, up to a unitary equivalence, we have : $\mathfrak{H}=L^{2}(\mathbb{R}, d \mu), \Omega$ is the constant function equal to 1 on $\mathbb{R}$ and $\pi(X)$ is the multiplication by the identity mapping of $\mathbb{R}$ on itself. Clearly the problem is determined ( $\Leftrightarrow \mu$ unique) if and only if $(\mathfrak{H}, \pi(X), \Omega)$ is unique up to a unitary equivalence under the above conditions.

Let $\left(\pi_{\phi}, \mathfrak{H}_{\phi}, D_{\phi}, \Omega_{\phi}\right)$ be the unbounded cyclic *-representation associated with $\phi$ $[1,2]$; we have canonically $\Omega_{\phi}=\Omega, D_{\phi}=$ linear hull of $\left\{\pi(X)^{n} \Omega\right\} \mathfrak{H}_{\phi}=$ closure of $D_{\phi}$ in $\mathfrak{H}$ and $\pi_{\phi}(X)=\pi(X) \nmid D_{\phi}$. It is well known, and easy to see, that the moment problem is determined if $\pi_{\phi}(X)$ is an essentially self-adjoint operator in $\mathfrak{H}_{\phi}$. Then, the solution is given, of course, by $\mathfrak{H}=\mathfrak{H}_{\phi}$ and $\pi(X)=\pi_{\phi}(X)^{*}$. Furthermore, in this case all the $\pi_{\phi}\left(X^{n}\right)=\pi_{\phi}(X)^{n}$ are also essentially self-adjoint operators $(n \in \mathbb{N})$.

In this paper, this type of result connecting determination and self-adjointness will be generalized. According to Part I, the $m$-problem on a *algebra $\mathfrak{A}$ is a generalization of the classical moment problem in the following sense: If $\mathfrak{U}$ is the algebra $\mathbb{C}\left[X_{1}, \ldots, X_{n}\right]$ of the polynomials with respect to $n$ indeterminates, then the $m$-problem on $\mathfrak{U}$ is exactly the $n$-dimensional classical moment problem.

It will be a consequence of the results of this paper that if $\phi$ is a strongly positive linear form on a *-algebra $\mathfrak{A}$ (i.e. a positive linear form for which the $m$-problem is soluble [1]) and if $\pi_{\phi}(h)$ is an essentially self-adjoint operator for any hermitian $h$ in $\mathfrak{U}$, then the $m$-problem is determined. However, in the applications, it is generally too strong to assume that $\pi_{\phi}(h)$ is essentially self-adjoint for all the $h=h^{*}$ in $\mathfrak{A}$. What frequently happens is that essential self-adjointness holds for the $\pi_{\phi}(h)$ when $h$ runs over a generating subset $\Sigma$ of hermitian elements of $\mathfrak{A}$, but in contrast to what happens when $\mathfrak{A}=\mathbb{C}[X]$, this does not imply, in general, essential self-adjointness for all the $\pi_{\phi}(h)$ with $h=h^{*}$ in $\mathfrak{A}$.

For instance, let $\mathfrak{A}$ be the tensor algebra $T\left(\mathbb{C}^{2}\right)$ over $\mathbb{C}^{2}$ equipped with the unique involution for which $x=\left(\begin{array}{l}1 \\ 0\end{array}\right)$ and $y=\left(\begin{array}{l}0 \\ 1\end{array}\right)\left(\in \mathbb{C}^{2} \subset T\left(\mathbb{C}^{2}\right)\right)$ are hermitian and let $\phi$ be the linear form on $\mathfrak{A}$ defined by:

$$
\begin{aligned}
\phi\left(\left(\lambda_{1} x+\mu_{1} y\right) \ldots\left(\lambda_{n} x+\mu_{n} y\right)\right)= & \pi^{-1 / 2} \int_{\mathbb{R}} d q e^{-q^{2} / 2}\left(\lambda_{1} q-\mu_{1} i \frac{d}{d q}\right) \ldots \\
& \left(\lambda_{n} q-\mu_{n} i \frac{d}{d q}\right) e^{-q^{2} / 2} .
\end{aligned}
$$

$\phi$ is a positive linear form on $\mathfrak{A}$ and since $\mathfrak{A}$ is a tensor algebra over an involutive space, we know (see Part I, Section 4, Theorem 2) that $\phi$ is strongly positive so the $m$ problem is soluble. On the other hand, we have canonically $\mathfrak{H}_{\phi}=L^{2}(\mathbb{R}, d q), \Omega_{\phi}$ $=\pi^{-1 / 4} e^{-q^{2} / 2}, \quad D_{\phi}=\left\{P(q) e^{-q^{2} / 2} \mid P(X) \in \mathbb{C}[X]\right\}, \quad\left(\pi_{\phi}(x) \Psi\right)(q)=q \Psi(q) \quad$ and $\left(\pi_{\phi}(y) \Psi\right)(q)=-i \frac{d \Psi}{d q}(q)$ for $\Psi \in D_{\phi}$. It is well known that $\pi_{\phi}(x)$ and $\pi_{\phi}(y)$ are essentially self-adjoint operators but it is also known ${ }^{1}$ that there are hermitian combinations of $\pi_{\phi}(x)$ and $\pi_{\phi}(y)$ which are not essentially self-adjoint on $D_{\phi}$; in other words there are hermitian elements $h$ of $\mathfrak{A}$ for which $\pi_{\phi}(h)$ are not essentially

$1 \quad \pi_{\phi}(x)^{n} \pi_{\phi}(y) \pi_{\phi}(x)^{n}, n \geqq 2$ 
self-adjoint operators so here $\Sigma=\{x, y\}$. In fact $\Omega_{\phi}$ is an entire vector for $\pi_{\phi}(t x+r y)$, $\forall t, r \in \mathbb{R}$, so one may take $\Sigma=\{t x+r y \mid t, r \in \mathbb{R}\}=E_{2} \cong \mathbb{R}^{2}$ as well as $\Sigma=\{x, y\}$ (=basis of $E_{2}$ ). It is worth noticing here that if $\dot{\phi}$ denotes the positive linear form on the quotient algebra $\mathfrak{\mathfrak { U }}=\mathfrak{U} / \pi_{\phi}^{-1}(0)$ induced by $\phi, \dot{\phi}$ is not strongly positive; indeed $\dot{\mathfrak{U}}$ is the *-algebra generated by Heisenberg canonical commutation relations and it is well known that this algebra does only admit unbounded *-representations so there are no $C^{*}$-semi-norm on $\mathfrak{\mathfrak { U }}$ and therefore no non-trivial strongly positive linear form on $\mathfrak{\mathfrak { A }}$. The situation is similar if one considers the algebra generated by the free hermitian field and this phenomena has been already pointed out in the Part I of this work [see the Remark 9b) in Part I].

In Part I of this work, we have associated to any *-algebra $\mathfrak{A}$ with a unit a $C^{*}$ algebra $\mathfrak{B}(\mathfrak{U}) . \mathfrak{B}(\mathfrak{U})$ is such that any hermitian element $h$ of $\mathfrak{U}$ determines a *homomorphism $f \mapsto f(h)$ of the $C^{*}$-algebra $\mathscr{C}_{(0)}(\mathbb{R})$ of complex continuous functions vanishing at infinity on $\mathbb{R}$ into $\mathfrak{B}(\mathfrak{U})$. Furthermore, the ranges of these homorphisms generate $\mathfrak{B}(\mathfrak{A})$ as $C^{*}$-algebra (when $h$ runs over the set $\mathfrak{U}^{h}$ of all the hermtian elements of $\mathfrak{U}$ ) (see Part I, Section 6). Let $\Sigma$ be a subset of $\mathfrak{U}^{h}$ and let $\mathfrak{B}(\Sigma)$ be the $C^{*}$ subalgebra of $\mathfrak{B}(\mathfrak{U})$ generated by $\left\{f(h) \mid h \in \Sigma, f \in \mathscr{C}_{(0)}(\mathbb{R})\right\}$. In this paper, we shall show the following. If $\phi$ is a strongly positive linear form on $\mathfrak{A}$, if $\left(\pi_{\phi}, \mathfrak{H}_{\phi}, D_{\phi}, \Omega_{\phi}\right)$ is the associated cyclic *-representation and if $\Sigma$ is a generating subset of hermitian elements of $\mathfrak{A}$ then, any solution $\omega$ of the $m$-problem for $\phi[\omega$ is a positive linear form on $\mathfrak{B}(\mathfrak{U})$, see Section 7 in Part I] leads to a cyclic representation $\pi_{\Sigma}$ of $\mathfrak{B}(\Sigma)$ in a Hilbert space $\mathfrak{H}_{\Sigma}$ which contains $\mathfrak{H}_{\phi}$ as closed subspace and with $\Omega_{\phi}$ as cyclic vector such that $\omega(f(h))=\left(\Omega_{\phi} \mid \pi_{\Sigma}(f(h)) \Omega_{\phi}\right) ; h \in \Sigma, f \in \mathscr{C}_{(0)}(\mathbb{R})$. Furthermore, if the $\pi_{\phi}(h)$ are essentially self-adjoint when $h \in \Sigma$ then $\mathfrak{H}_{\Sigma}=\mathfrak{H}_{\phi}, \pi_{\Sigma}$ is unique and we have: $\pi_{\Sigma}(f(h))$ $=f\left(\overline{\pi_{\phi}(h)}\right) ; \forall h \in \Sigma, \forall f \in \mathscr{C}_{(0)}(\mathbb{R})$. In any case, $\left(\pi_{\Sigma}, \mathfrak{H}_{\Sigma}, \Omega_{\phi}\right)$ is canonically the G.N.S. triplet associated with $\omega \uparrow \mathfrak{B}(\Sigma)$ and $\phi$ can be reconstructed from $\omega \uparrow \mathfrak{B}(\Sigma)$.

For instance, let $\mathfrak{U}, x, y$, and $\phi$ be as in the above example and let $\Sigma=\{x, y\}$. Then there is a unique representation $\pi_{\Sigma}$ of $\mathfrak{B}(\{x, y\})=\mathfrak{B}(\Sigma)$ in $\mathfrak{H}_{\phi}$ for which $\Omega_{\phi}$ is cyclic and $\left.\pi_{\Sigma}(f(x))=f\left(\overline{\pi_{\phi}(x)}\right), \pi_{\Sigma}(f(y))=f \overline{\left(\pi_{\phi}(y)\right.}\right)$ for any $f \in \mathscr{C}_{(0)}(\mathbb{R})$.

For the applications to quantum field theory, it is important to realize that, following H. J. Borchers, we consider that a (scalar hermitian) quantum field is a *representation of the tensor algebra over the space of complex test functions. Furthermore, we shall be interested in self-adjointness properties of the field operator, so we take $\mathfrak{A}=T(\mathscr{D}(M))[=$ the tensor algebra over the space $\mathscr{D}(M)$ of all complex $C^{\infty}$ functions with compact support on the space-time $\left.M\right]$ and $\Sigma=\mathscr{D}(M$, $\mathbb{R})=$ real $C^{\infty}$ functions with compact supports. It turns out that $\mathfrak{B}(\Sigma)$ is the quasilocalizable $C^{*}$-algebra $\hat{\mathfrak{B}}(M)$ introduced in Part $\mathrm{I}$.

We use the above results to show that the bounded observables of cut-off field theories generate concrete $C^{*}$-algebras which are images of cyclic representations of the (universal) quasi-localizable $C^{*}$-algebra $\hat{\mathfrak{B}}(M)$ with the ground states of the hamiltonians as cyclic vectors. The corresponding states on $\hat{\mathfrak{B}}(M)$ being elements of the weakly compact states' space of $\hat{\mathfrak{B}}(M)$, a program of removing the cut-offs by compactness arguments is suggested. A difficulty connected with general features of the construction described in the work (Part I and this paper) is pointed out: Namely we do not take into account the topologies of our basic spaces (*-algebras, spaces of test functions) with this algebraical construction. 
In a forthcoming paper, we shall analyze the restrictions which come from the continuity properties in the $m$-problem.

In Section 2 we analyse the connexion between the determination of the $m$ problem and the self-adjointness properties in the corresponding hermitian cyclic representation $[1,2]$. We give a generalization of the well-known connexion [3-5] between the determination of the measure in the classical moment problem and the self-adjointness properties of the polynomials as operators in the corresponding $L^{2}$ space.

In Section 3 we discuss the dependence of the problem on the choice of sets of $C^{*}$-semi-norms. We introduce a notion which generalize the notion of support in the classical moment problem.

In Section 4 the action of *-homomorphisms is investigated. We define $C^{*}$ algebras associated with real vector spaces. This generalizes the definition of the quasi-localizable $C^{*}$-algebra given in Part I.

In Section 5, we apply the above results to quantum field theory. We show that, if for every real test function the smeared field operator is essentially self-adjoint on the cyclic subspace generated from a unit vector in Hilbert space by the polynomial algebra of the smeared field operators, then the $m$-problem for the corresponding vector state on the tensor algebra over the space of test functions is determined on the quasi-localizable $C^{*}$-algebra and that the corresponding representation of the quasi-localizable $C^{*}$-algebra generates the bounded observables of the field.

The last result works for a hermitian scalar field in a very general sense. In particular, it is pointed out in Section 6 that a form of $\phi^{4}$-cut-off field introduced by Jaffe in his thesis [6] fall in this class. The self-adjointness of the space-time smeared cut-off field is obtained as an application of a general method introduced by Glimm and Jaffe in their study of $\left(\phi^{4}\right)_{2}$ theory [15] (see also [16] and the paper of McBryan [17]). We define, for each cut-off, a state on the quasi-localizable $C^{*}$-algebra which correspond to the ground-state-expectation-values of the bounded observables. We allow the mass, the coupling constant and the field normalization to vary with the value of the cut-off.

In conclusion, we discuss some possible pathologies associated with the "limits" obtained (by compactness) when the cut-off is removed. These pathologies were avoided in the work of Glimm and Jaffe on $\left(\phi^{4}\right)_{2}$ when they proved the "local normality" of the "vacuum state" (see, for instance, the Theorem 4.2.1 in Les Houches 1970, [18]).

Throughout this paper, we use the notation of Part I. If $\mathfrak{B}$ is a $C^{*}$-algebra and if $\omega$ is a positive linear form on $\mathfrak{B}$, we use the term, G.N.S. triplet associated with $\omega$ to denote triplet $\left(\pi_{\omega}, \mathfrak{H}_{\omega}, \Omega_{\omega}\right)$ of the cyclic representation $\pi_{\omega}$ in Hilbert space $\mathfrak{H}_{\omega}$ with cyclic vector $\Omega_{\omega}$ obtained from $\omega$ by the Gelfand-Naimark-Segal construction (G.N.S. construction).

$\mathscr{D}_{(0)}\left(\mathbb{R}^{n}\right)$ denote the space of continuous complex functions with compact supports on $\mathbb{R}^{n}$.

$\mathscr{D}\left(\mathbb{R}^{n}\right)$ denote the space of $C^{\infty}$-complex functions with compact supports on $\mathbb{R}^{n}$.

$\mathscr{C}\left(\mathbb{R}^{n}\right)$ is the space of complex continuous functions on $\mathbb{R}^{n}$. on $\mathbb{R}^{n}$.

$\mathscr{C}_{(0)}\left(\mathbb{R}^{n}\right)$ is the $C^{*}$-algebra of complex continuous functions vanishing a infinity 


\section{Self-Adjointness and Determination}

Throughout this section $\mathfrak{A}$ is a *algebra with a unit $(\mathbb{1} \in \mathfrak{A}), \Gamma$ is a separating directed set of $C^{*}$-semi-norms on $\mathfrak{A}$ and $\mathfrak{B}(\mathfrak{A}, \Gamma)$ is the associated $C^{*}$-algebra defined in Part I (Part I, Section 6, Definition 3).

Lemma 1. Let $\pi$ be a representation of $\mathfrak{B}(\mathfrak{A}, \Gamma)$ in a Hilbert space $\mathfrak{H}_{\pi}$ and let $h$ be an arbitrary hermitian element of $\mathfrak{A}$. Then, in the Hilbert subspace $\mathfrak{H}_{\pi}(h)$ of $\mathfrak{H}_{\pi}$ spanned by the set $\left\{\pi(f(h)) \Phi \mid \Phi \in \mathfrak{H}_{\pi}\right.$ and $\left.f \in \mathscr{C}_{(0)}(\mathbb{R})\right\}$, there is a unique self-adjoint operator $\pi(h)$ for which we have: $\pi(h) \pi(f(h)) \Phi=\pi\left(f^{I}(h)\right) \Phi, \forall \Phi \in \mathfrak{H}_{\pi}$ and $\forall f \in \mathscr{D}_{(0)}(\mathbb{R})$, where $f^{I} \in \mathscr{D}_{(0)}(\mathbb{R})$ is defined by $f^{I}(t)=t f(t) \quad(\forall t \in \mathbb{R})$. Furthermore, we have $\pi(f(h)) \mid \mathfrak{H}_{\pi}(h)=f(\pi(h)), \forall f \in \mathscr{C}_{(0)}(\mathbb{R})$, and if $A$ is a bounded operator in $\mathfrak{H}_{\pi}$ which commutes with $\pi(f(h))$ for any $f \in \in \mathscr{C}_{(0)}(\mathbb{R})$ then $\mathfrak{G}_{\pi}(h)$ is stable by $A$ and the restriction of $A$ to $\mathfrak{H}_{\pi}(h)$ commutes with $\pi(h)$ (i.e. its spectral projections).

Proof $^{2}$. Let $D_{\pi}^{0}(h)$ be the linear hull in $\mathfrak{H}_{\pi}$ of the set $\left\{\pi(f(h)) \Phi \mid \Phi \in \mathfrak{H}_{\pi}\right.$ and $\left.f \in \mathscr{D}_{(0)}(\mathbb{R})\right\} ; D_{\pi}^{0}(h)$ is a dense subspace of $\mathfrak{G}_{\pi}(h)$ and there is a unique linear mapping $\pi^{0}(h)$ of $D_{\pi}^{0}(h)$ into itself satisfying $\pi^{0}(h) \pi(f(h)) \Phi=\pi\left(f^{I}(h)\right) \Phi, \quad \forall \Phi \in \mathfrak{H}_{\pi}$ and $\forall f \in \mathscr{D}_{(0)}(\mathbb{R})$. As an operator in $\mathfrak{H}_{\pi}(h), \pi^{0}(h)$ is a symmetric operator for which $D_{\pi}^{0}(h)$ is a dense domain of entire vectors. Therefore $\pi^{0}(h)$ is closable and its closure $\pi(h)$ is a self-adjoint operator in $\mathfrak{H}_{\pi}(h)$ which is clearly unique under the above conditions. Using the definition and the fact that $D_{\pi}^{0}(h)$ is dense stable domain of entire and even bounded vectors for $\pi(h)$, it is straightforward to see that the equality $\pi(f(h)) \Phi$ $=f(\pi(h)) \Phi$ holds for any $f \in \mathscr{D}_{(0)}(\mathbb{R})$ and any $\Phi \in D_{\pi}^{0}(h)$ and therefore, by continuity it also holds for $f \in \mathscr{C}_{(0)}(\mathbb{R})$ and $\Phi \in \mathfrak{G}_{\pi}(h)$.

Suppose that $A \in \mathscr{L}\left(\mathfrak{H}_{\pi}\right)$ commutes with $\left\{\pi(f(h)) \mid f \in \mathscr{C}_{(0)}(\mathbb{R})\right\}$. Then, for any $\Phi \in \mathfrak{H}_{\pi}$ and for any $f \in \mathscr{D}_{(0)}(\mathbb{R})$, we have: $A \pi(f(h)) \Phi \in D_{\pi}^{0}(h)$, since $A \pi(f(h)) \Phi=\pi(f(h)) A \Phi$, and, $A \pi(h) \pi(f(h)) \Phi=A \pi\left(f^{1}(h)\right) \Phi=\pi\left(f^{1}(h)\right) A \Phi=\pi(h)$ $A \pi(f(h)) \Phi$. Therefore we have: $A D_{\pi}^{0}(h) \subset D_{\pi}^{0}(h)$ and $A \pi(h) \Phi=\pi(h) A \Phi$ for any $\Phi \in D_{\pi}^{0}(h)$. Let $\Phi_{\alpha}$ be a net of vectors in $D_{\pi}^{0}(h)$ such that $\left(\Phi_{\alpha}\right)$ converges to $\Phi$ and $\left(\pi(h) \Phi_{\alpha}\right)$ converges to $\Psi$ in $\mathfrak{H}_{\pi}(h)$; then $A \Phi=\lim A \Phi_{\alpha}\left(\in \mathfrak{H}_{\pi}(h)\right)$ and we also have $A \Psi=\lim \left(A \pi(h) \Phi_{\alpha}\right)=\lim \left(\pi(h) A \Phi_{\alpha}\right)$ (since $A$ is bounded). So, if $D_{\pi}(h)$ denote the domain of $\pi(h) \quad\left(D_{\pi}^{0}(h) \subset D_{\pi}(h) \subset \mathfrak{H}_{\pi}(h)\right)$, we have: $A D_{\pi}(h) \subset D_{\pi}(h)$ and $A \pi(h) \Phi=\pi(h) A \Phi, \forall \Phi \in D_{\pi}(h)$.

This achieves the proof of the Lemma 1.

Let $\phi$ be a positive linear form on $\mathfrak{A}$ and let $\pi_{\phi}$ be the corresponding cyclic *representation [8] in Hilbert space $\mathfrak{H}_{\phi}$ with cyclic vector $\Omega_{\phi} \in \mathfrak{H}_{\phi}$ and domain $D_{\phi}=\pi_{\phi}(\mathfrak{I}) \Omega_{\phi}$ such that $\phi(x)=\left(\Omega_{\phi} \mid \pi_{\phi}(x) \Omega_{\phi}\right)$; it is well known that, for $\phi$ fixed, $\left(\pi_{\phi}\right.$, $\mathfrak{H}_{\phi}, D_{\phi}$ ) is unique up to a unitary equivalence under the above conditions. Suppose that $\phi$ is $\Gamma$-strongly positive and let $\omega$ be a solution of the $m(\Gamma)$-problem for $\phi$. Let $\left(\pi_{\omega}, \mathfrak{H}_{\omega}, \Omega_{\omega}\right)$ be the G.N.S. triplet associated with $\omega$ and let us use the notation of the Section 7 in Part I (in particular see the Proposition 3 in Part I). In the Proposition 3 of Part I, it was shown that $\pi_{\phi}(x) \Omega_{\vec{\phi}} \Psi_{\omega}(x)$ define an isometric inbedding of $\mathfrak{H}_{\phi}$ in $\mathfrak{H}_{\omega}$. Therefore it is justified, and we shall always do so in the following, to identify $\mathfrak{H}_{\phi}$ with a closed subspace of $\mathfrak{H}_{\omega}$, writing: $\Omega_{\phi}=\Omega_{\omega}, \pi_{\phi}(x) \Psi_{\omega}(y)=\Psi_{\omega}(x y) ; \forall x, y \in \mathfrak{A}$ [whenever $\omega$ is a solution of the $m(\Gamma)$-problem for $\phi$ ]. In general, the inclusion $\mathfrak{S}_{\phi} \subset \mathfrak{S}_{\omega}$ is strict (see what happens in the classical moment problem).

2 This proof is influenced by the appendix of a paper of Ruelle [7] 
Lemma 2. Let $\phi$ be a $\Gamma$-strongly positive linear form on $\mathfrak{A}$ and let $\omega$ be an arbitrary solution of the $m(\Gamma)$-problem for $\phi$. Then we have: $D_{\phi}=\Psi_{\omega}(\mathfrak{A}) \subset D_{\pi_{\omega}}(h)$ and $\pi_{\phi}(h)=\pi_{\omega}(h) \uparrow D_{\phi}, \forall h=h^{*} \in \mathfrak{U}$ (where $D_{\pi_{\omega}}(h)$ denote the domain of the self-adjoint operator $\pi_{\omega}(h)$ in $\mathfrak{H}_{\pi_{\omega}}(h)$ defined as in Lemma 1 and where we make the above identifactions).

Proof. Let $\Psi_{\omega}(x)(x \in \mathfrak{A})$ be an arbitrary element of $D_{\phi}$. We know (Proposition 3 in Part I, Section 7) that $\omega_{x}$ is a solution of the $m(\Gamma)$-problem for $\phi_{x}$, where $\omega_{x}$ and $\phi_{x}$ are defined by: $\omega_{x}(y)=\left(\Psi_{\omega}(x) \mid \pi_{\omega}(y) \Psi_{\omega}(x)\right), \forall y \in \mathfrak{B}(\mathfrak{U}, \Gamma), \phi_{x}(y)=\phi\left(x^{*} y x\right)$ $=\left(\Psi_{\omega}(x) \mid \pi_{\phi}(y) \Psi_{\omega}(x)\right), \forall y \in \mathfrak{U}$.

It follows (see, in Part I, the Theorems 4 and 5) that the positive measure $\mu_{h, \omega_{x}}$ on $\mathbb{R}$ defined by $\left(h=h^{*} \in \mathfrak{N}\right)$

$$
\omega_{x}(f(h))=\int f(t) d \mu_{h, \omega_{x}}(t), \forall f \in \mathscr{C}_{(0)}(\mathbb{R}),
$$

is a rapidly decreasing measure and that we have:

$$
\phi_{x}\left(h^{n}\right)=\int t^{n} d \mu_{h, \omega_{x}}(t), \forall n \in \mathbb{N} .
$$

Let $\chi_{n}$ be a sequence of continuous function on $\mathbb{R}$ such that $0 \leqq \chi_{n} \leqq 1, \chi_{n}(t)=1$ if $|t|$ $<n, \chi_{n}(t)=0$ if $|t| \geqq n+1$. Then $\pi_{\omega}\left(\chi_{n}(h)\right) \Psi_{\omega}(x)$ is a sequence of element of $D_{\pi_{\omega}}^{0}(h)$ and $\pi_{\omega}(h) \pi_{\omega}\left(\chi_{n}(h)\right) \Psi_{\omega}(x)=\pi_{\omega}\left(\chi_{n}^{I}(h)\right) \Psi_{\omega}(x)$ (with the notation of Lemma 1). We have:

$$
\begin{aligned}
\left\|\pi_{\phi}(h) \Psi_{\omega}(x)-\pi_{\omega}(h) \pi_{\omega}\left(\chi_{n}(h)\right) \Psi_{\omega}(x)\right\|^{2} & =\int t^{2}\left(1-\chi_{n}(t)\right)^{2} d \mu_{h, \omega_{x}}(t) \\
\left\|\Psi_{\omega}(x)-\pi_{\omega}\left(\chi_{n}(h)\right) \Psi_{\omega}(x)\right\|^{2} & =\int\left(1-\chi_{n}(t)\right)^{2} d \mu_{h, \omega_{x}}(t) .
\end{aligned}
$$

Both terms converge to zero when $n$ goes to infinity; so $\Psi_{\omega}(x) \in D_{\pi_{\omega}}(h)$ and $\pi_{\omega}(h) \Psi_{\omega}(x)=\pi_{\phi}(h) \Psi_{\omega}(x)$. This prove the lemma since $x \in \mathfrak{U}$ and $h=h^{*} \in \mathfrak{A}$ are arbitrary.

Corollary 1. Let $\phi$ and $\omega$ be as in Lemma 2 and let $h=h^{*}$ be a hermitian element of $\mathfrak{U}$ such that we have: $\pi_{\omega}(f(h)) \mathfrak{H}_{\phi} \subset \mathfrak{H}_{\phi}, \forall f \in \mathscr{C}_{(0)}(\mathbb{R})$. Then $\pi_{\omega}(h) \uparrow\left(\mathfrak{H}_{\phi} \cap D_{\pi_{\omega}}(h)\right)$ is a selfadjoint extension of the symmetric operator $\pi_{\phi}(h)$ in $\mathfrak{S}_{\phi}$.

Corollary 2. Let $\phi$ and $\omega$ be as above, let $\Sigma$ be a set of hermitian elements of $\mathfrak{A}$ which generates $\mathfrak{U}$ and let $\mathfrak{B}(\Sigma, \Gamma)$ denote the $C^{*}$-subalgebra of $\mathfrak{B}(\mathfrak{U}, \Gamma)$ generated by the set $\left\{f(h) \mid h \in \sum\right.$ and $\left.f \in \mathscr{C}_{(0)}(\mathbb{R})\right\}$. Then we have $\mathfrak{H}_{\phi} \subseteq \pi_{\omega}(\mathfrak{B}(\Sigma, \Gamma)) \Omega_{\omega}$ (where the bar denote the closure in $\left.\mathfrak{H}_{\omega}\right)$ and if the equality holds $\pi_{\omega}(h) \uparrow\left(\mathfrak{H}_{\phi} \cap D_{\pi_{\omega}}(h)\right)$ is a self-adjoint extension of $\pi_{\phi}(h), \forall h \in \Sigma$ (these corollaries need not to be proved).

Theorem 1. Let $\phi$ be a $\Gamma$-strongly positive linear form on $\mathfrak{U}$ and let $\Sigma$ be a set of hermitian elements of $\mathfrak{A}$. Suppose that for any $h \in \Sigma, \pi_{\phi}(h)$ is an essentially self-adjoint operator in $\mathfrak{H}_{\phi}$. Then the $m(\Gamma)$-problem for $\phi$ is determined on the $C^{*}$-subalgebra $\mathfrak{B}(\Sigma, \Gamma)$ of $\mathfrak{B}(\mathfrak{U}, \Gamma)$ generated by the set $\left\{f(h) \in \mathfrak{B}(\mathfrak{U}, \Gamma) \mid h \in \Sigma\right.$ and $\left.f \in \mathscr{C}_{(0)}(\mathbb{R})\right\}$. Furthermore, if $\omega$ is an arbitrary solution of the $m(\Gamma)$-problem for $\phi$ and if $\overline{\pi_{\phi}(h)}$ denote the closure of $\pi_{\phi}(h)$, for $h \in \Sigma$ (considered as an operator in $\mathfrak{H}_{\phi}$ ), we have: $\left.\pi_{\omega}(f(h)) \uparrow \mathfrak{H}_{\phi}=f \overline{\left(\pi_{\phi}(h)\right.}\right), \forall f \in \mathscr{C}_{(0)}(\mathbb{R})$. 
Proof. It follows from the Lemma 2 that, for any hermitian element $h$ of $\mathfrak{A}$, we have $\left.\operatorname{dom} \overline{\left(\pi_{\phi}(h)\right.}\right) \subset D_{\pi_{\omega}}(h)$ and $\overline{\pi_{\phi}(h)}=\pi_{\omega}(h) \uparrow \operatorname{dom} \overline{\left(\pi_{\phi}(h)\right)}$ for any solution $\omega$ of the $m(\Gamma)$ problem for $\phi$. If $h \in \Sigma, \overline{\pi_{\phi}(h)}$ is by assumption a self-adjoint operator in $\mathfrak{H}_{\phi}$; let $t \mapsto E_{h}(t)$ be its spectral resolution $\overline{\left(\pi_{\phi}(h)\right.}=\int t d E_{h}(t)$ in $\left.\mathfrak{H}_{\phi}\right)$. For any positive number $s$, let $\mathfrak{G}_{s}(h)$ be the closed subspace of $\mathfrak{H}_{\phi}$ defined by: $\mathfrak{H}_{s}(h)=\int_{-s}^{+s} d E_{h}(t) \mathfrak{G}_{\phi} . \bigcup_{s \geqq 0} \mathfrak{H}_{s}(h)$ is a dense subspace of $\mathfrak{H}_{\phi}$ contained in the domain $\left.\operatorname{dom} \frac{-s}{\left(\pi_{\phi}(h)\right.}\right)$ of the self-adjoint operator $\overline{\pi_{\phi}(h)}$ in $\mathfrak{S}_{\phi}$. We have $\overline{\pi_{\phi}(h)} \mathfrak{G}_{s}(h) \subset \mathfrak{G}_{s}(h)$ and $\pi_{\omega}(h)\left\lceil\mathfrak{S}_{s}(h)=\overline{\pi_{\phi}(h)} \uparrow \mathfrak{G}_{s}(h)\right.$ so $\pi_{\omega}(f(h))\left\lceil\mathfrak{H}_{s}(h)=f\left(\pi_{\phi}(h)\right) \uparrow \mathfrak{G}_{s}(h) \forall f \in \mathscr{C}_{(0)}(\mathbb{R})\right.$ [since $\overline{\pi_{\phi}(h)} \uparrow \mathfrak{G}_{s}(h)$ is a bounded selfadjoint operator in $\mathfrak{H}_{s}(h)$ with $\left.\left\|\overline{\pi_{\phi}(h)} \uparrow \mathfrak{H}_{s}(h)\right\| \leqq s\right]$. So we also have: $\pi_{\omega}(f(h)) \uparrow \mathfrak{H}_{\phi}$ $=f \overline{\left(\pi_{\phi}(h)\right)}, \forall f \in \mathscr{C}_{(0)}(\mathbb{R})$ and $\forall h \in \Sigma$ [where $\omega$ is an arbitrary solution of the $m(\Gamma)$ problem for $\phi]$. This implies in particular that if $\omega_{1}$ and $\omega_{2}$ are two solutions of the $m(\Gamma)$-problem then we have:

$$
\pi_{\omega_{1}}(x) \Phi=\pi_{\omega_{2}}(x) \Phi \in \mathfrak{H}_{\phi}, \forall \Phi \in \mathfrak{H}_{\phi} \text { and } \forall x \in \mathfrak{B}(\Sigma, \Gamma) .
$$

Therefore (since $\Omega_{\omega_{1}}=\Omega_{\omega_{2}}=\Omega_{\phi} \in \mathfrak{G}_{\phi}$ ) we have, $\forall x \in \mathfrak{B}(\Sigma, \Gamma) \omega_{1}(x)=\omega_{2}(x)$. This achieves the proof of the theorem.

Remark 1. a) Replacing $\Sigma$ by $\Sigma \cup\{\mathbb{1}\}$ it follows that we may replace in the statement $\mathfrak{B}(\Sigma, \Gamma)$ by $\mathfrak{B}(\Sigma \cup\{\mathbb{1}\}, \Gamma)$ [which contains the identity of $\mathfrak{B}(\mathfrak{H}, \Gamma)]$.

b) Remembering that if $\Gamma_{M}$ is the set of all the $C^{*}$-semi-norms on $\mathfrak{A}$ then $\mathfrak{B}\left(\mathfrak{A}, \Gamma_{M}\right)$ is denoted by $\mathfrak{B}(\mathfrak{U})$ (and called the $C^{*}$-algebra associated to $\mathfrak{A}$ ), the $m\left(\Gamma_{M}\right)$-problem is called the $m$-problem and a $\Gamma_{M}$-strongly positive linear form is called a strongly positive linear form and that, furthermore, every solution of the $m(\Gamma)$-problem is a fortiori (canonically) a solution of the $m$-problem for $\phi$ [via the canonical surjective ${ }^{*}$-homomorphism: $\left.\mathfrak{B}(\mathfrak{U}) \mapsto \mathfrak{B}(\mathfrak{H}, \Gamma)\right]$; it follows that the condition of Theorem 1 is already a sufficient condition for the determination of the $m$-problem. Since it may happen that a $m(\Gamma)$-problem is determined and that there are several solutions of the corresponding $m$-problem (compare Stieltjes problem and Hamburger's problem in the classical moment problem), one cannot expect, in general, that the condition of Theorem 1 is a necessary condition of determination of the $m(\Gamma)$-problem.

c) In the proof of theorem it was shown that the representation $\pi_{\omega}$ of $\mathfrak{B}(\Sigma, \Gamma)$ leaves $\mathfrak{H}_{\phi}$ invariant and is unique on $\mathfrak{G}_{\phi}$ [i.e. independent of the choice of the solution $\omega$ of the $m(\Gamma)$-problem and even the $m$-problem by b), replacing $\mathfrak{B}(\Sigma, \Gamma)$ by $\mathfrak{B}(\Sigma)]$. Finally, let us notice that this implies that, if $\Sigma$ generates $\mathfrak{A}$, the closure of $\pi_{\omega}(\mathfrak{B}(\Sigma, \Gamma)) \Omega_{\omega}$ in $\mathfrak{G}_{\omega}$ is exactly $\mathfrak{H}_{\phi}$ (compare with Corollary 2 ).

Lemma 3. Let $\phi$ be a pure state $e^{3}$ on $\mathfrak{A}$ which is $\Gamma$-strongly positive and let $\mathfrak{\Im}_{\phi}$ be the (convex and weakly compact) set of all the solutions of the $m(\Gamma)$-problem for $\phi$. Then every extreme point of $\mathfrak{\subseteq}_{\phi}$ is a pure state on $\mathfrak{B}(\mathfrak{H}, \Gamma)$.

Proof. Let $\omega$ be an extreme point of $\mathfrak{S}_{\phi}$ and let $\omega_{1}$ be a positive linear form on $\mathfrak{B}(\mathfrak{A}, \Gamma)$ such that $\omega \geqq \omega_{1}$. If $\pi_{\omega}$ is the cyclic representation associated with $\omega$, we

3 A state on $\mathfrak{A}$ is a positive linear form on $\mathfrak{A}$ for which $\phi(\mathbb{1})=1$. A positive linear form $\phi$ is pure if the only positive linear forms $\Psi$ satisfying $\phi\left(x^{*} x\right) \geqq \Psi\left(x^{*} x\right)$ are the multiples $\Psi=\lambda \phi$ of $\phi(0 \leqq \lambda \leqq 1)$ 
know that there is a positive $A_{1} \in \pi_{\omega}(\mathfrak{B}(\mathfrak{A}, \Gamma))^{\prime}$ with $0 \leqq A_{1} \leqq \mathbb{1}$ for which $\omega_{1}(x)$ $=\left(\Omega_{\omega} \mid A_{1} \pi_{\omega}(x) \Omega_{\omega}\right), \forall x \in \mathfrak{B}(\mathfrak{U}, \Gamma)$. From the last part of Lemma 1 and from Lemma 2 , it follows that we have $\phi_{1}(h)=\left(\Omega_{\omega} \mid A_{1} \pi_{\omega}(h) \Omega_{\omega}\right)=\left(\Omega_{\omega} \mid \pi_{\omega}(h) A_{1} \Omega_{\omega}\right)=\left(\Omega_{\phi} \mid A_{1} \pi_{\phi}(h) \Omega_{\phi}\right)$ $=\left(\pi_{\phi}(h) \Omega_{\omega} \mid A_{1} \Omega_{\phi}\right), \forall h \in \mathfrak{U}$, and from $0 \leqq A_{1} \leqq \mathbb{1}$ it follows that $\phi_{1}$ defines a positive linear form on $\mathfrak{A}$ for which $\phi_{1}\left(x^{*} x\right) \leqq \phi\left(x^{*} x\right)$. It follows that $\phi_{1}=\lambda \phi$. For some constant $0 \leqq \lambda \leqq 1$ (since $\phi$ is pure). This implies immediately that $\tilde{\omega}=\lambda^{-1} \omega_{1}$ is a solution of the $m(\Gamma)$-problem for $\phi$ if $\lambda \neq 0$, and that, if $\lambda \neq 1$ the same is true for $\tilde{\omega}_{1}^{\prime}$ $=\left(\omega-\omega_{1}\right) /(1-\lambda)$ so we have $\omega=\lambda \tilde{\omega}_{1}+(1-\lambda) \tilde{\omega}_{1}^{\prime}$ which implies (since $\omega$ is an extreme point in $\Im_{\phi}$ ) either $\tilde{\omega}_{1}=\tilde{\omega}_{1}^{\prime}=\omega$ either $\lambda=0$ or $\lambda=1$ so in any case $\lambda \omega_{1}=\omega$ which means that $\omega$ is pure.

Notice that, quite generally, if $\phi$ is an arbitrarily $\Gamma$-strongly positive linear form on $\mathfrak{U}$, if $\omega$ is a solution of the $m(\Gamma)$-problem for $\phi$ and if $P_{\phi}^{\omega}$ denote the orthogonal projection on $\mathfrak{H}_{\phi}$ (as a subspace of $\mathfrak{H}_{\omega}$ ) then the mapping $A \mapsto P_{\phi}^{\omega} A P_{\phi}^{\omega}$ of $\mathscr{L}\left(\mathfrak{H}_{\omega}\right)$ in $\mathscr{L}\left(\mathfrak{H}_{\phi}\right)$ maps the commutant $\pi_{\omega}(\mathfrak{B}(\mathfrak{H}, \Gamma))^{\prime}$ of $\pi_{\omega}(\mathfrak{B}(\mathfrak{U}, \Gamma))$ in the weak commutant $\pi_{\phi}(\mathfrak{U})_{w}^{\prime}$ of $\pi_{\phi}(\mathfrak{U})$ in $\mathfrak{H}_{\phi}$ (where $\pi_{\phi}$ is considered as a ${ }^{*}$-representation of $\mathfrak{U}$ in $\mathfrak{H}_{\phi}$ with domain $\left.D_{\phi}[2]\right)$.

Let $S(\mathfrak{A}, \Gamma)$ be the convex cone of all the $\Gamma$-strongly positive linear forms on $\mathfrak{A}$ and let $M(\mathfrak{U}, \Gamma)$ be the convex cone of all the positive linear forms on $\mathfrak{B}(\mathfrak{H}, \Gamma)$ which are solutions of $m(\Gamma)$-problems. Any element $\omega$ of $M(\mathfrak{A}, \Gamma)$ is solution of the $m(\Gamma)$ problem for a unique element $\phi_{\omega}$ of $S(\mathfrak{A}, \Gamma)$ and we have:

$$
\phi_{t_{1} \omega_{1}+t_{2} \omega_{2}}=t_{1} \phi_{\omega_{1}}+t_{2} \phi_{\omega_{2}} ; \quad \forall \omega_{1}, \omega_{2} \in M(\mathfrak{U}, \Gamma) \text { and } \forall t_{1}, t_{2} \in \mathbb{R}^{+} .
$$

Lemma 4. Let $\left(\phi_{\alpha}\right)$ be a net of $\Gamma$-strongly positive linear forms on $\mathfrak{A}$ and let us choose, for each $\alpha$, a solution $\omega_{\alpha}$ of the $m(\Gamma)$-problem for $\phi_{\alpha}$. Suppose that $\left(\phi_{\alpha}\right)$ converges weakly to $\phi$ (in the dual space of $\mathfrak{U}$ ) and let $\omega$ be the weak limit (in $\mathfrak{B}(\mathfrak{U}, \Gamma)^{\prime}$ ) of an arbitrary weakly convergent subnet of $\left(\omega_{\alpha}\right)$. Then $\omega$ is a solution of the $m(\Gamma)$-problem for $\phi$.

Proof. Let $\left(\omega_{\beta}\right)$ be a subnet of $\left(\omega_{\alpha}\right)$ which converges weakly to $\omega$. For each $\beta$ let $\psi_{\beta}$ be the linear form on the subspace $\mathfrak{U}+\mathfrak{B}(\mathfrak{U}, \Gamma)$ of $\mathscr{A}(\mathfrak{U}, \Gamma)$ which is positive on $(\mathfrak{U}$ $+\mathfrak{B}(\mathfrak{A}, \Gamma)) \cap \mathscr{A}^{+}(\mathfrak{U}, \Gamma)$ and satisfies : $\phi_{\beta}=\psi_{\beta} \uparrow \mathfrak{U}$ and $\omega_{\beta}=\psi_{\beta} \uparrow \mathfrak{B}(\mathfrak{A}, \Gamma)$. The net $\left(\psi_{\beta}\right)$ is by assumption simply convergent on $\mathfrak{A}$ and on $\mathfrak{B}(\mathfrak{A}, \Gamma)$ so it is simply convergent to a linear form $\psi$ on $\mathfrak{U}+\mathfrak{B}(\mathfrak{U}, \Gamma)$ which is positive on $(\mathfrak{U}+\mathfrak{B}(\mathfrak{U}, \Gamma)) \cap \mathscr{A}^{+}(\mathfrak{A}, \Gamma)$ and such that we have:

$$
\phi=\psi \uparrow \mathfrak{A} \quad \text { and } \quad \omega=\psi \uparrow \mathfrak{B}(\mathfrak{A}, \Gamma) .
$$

Remark 2. This lemma implies in particular that if the $m(\Gamma)$-problem for $\phi$ is determined on $\mathfrak{B}(\Sigma, \Gamma)$ (for some $\left.\Sigma \subset \mathfrak{U}^{k}\right)$ then the net $\omega_{\alpha} \uparrow \mathfrak{B}(\Sigma, \Gamma)$ is weakly convergent.

\section{Stability of the $m$-Problem: Supports}

Let $\mathfrak{U}$ be a *-algebra with a unit and let $\mathscr{N}(\mathfrak{U})$ be the set of all the $C^{*}$-semi-norms on $\mathfrak{A}$ equipped with the uniformity of the simple convergence on $\mathfrak{A}$. The relations caracterizing the $C^{*}$-semi-norms define a closed subset in $\mathbb{R}^{\mathfrak{A}}$ so $\mathscr{N}(\mathfrak{U})$ is a complete uniform space. Furthermore, $\mathscr{N}(\mathfrak{U})$ is canonically an ordered directed set. 
Let $\Gamma$ be a directed set of $C^{*}$-semi-norms on $\mathfrak{A}$ [i.e. $\Gamma$ is a directed subset of $\mathscr{N}(\mathfrak{A})]$ and let $\mathscr{T}_{\Gamma}$ be the locally convex topology on $\mathfrak{A}$ generated by $\Gamma$. According to Part I, the directed set $\tilde{\Gamma}$ of all the $\mathscr{T}_{\Gamma}$-continuous $C^{*}$-semi-norms on $\mathfrak{A}$ consists of all the $C^{*}$-semi-norms on $\mathfrak{A}$ which are bounded by $C^{*}$-semi-norms of $\Gamma$ and all the concepts and constructions introduced so far do only on $\tilde{\Gamma}(\mathscr{A}(\mathfrak{A}, \Gamma)=\mathscr{A}(\mathfrak{A}, \tilde{\Gamma})$. etc....). It follows that the convenient assumption that $\Gamma$ is directed may be dropped. Indeed, if $\Gamma$ is an arbitrary set of $C^{*}$-semi-norms $(\Gamma \subset \mathscr{N}(\mathfrak{A}))$ on $\mathfrak{U}$, then the locally convex topology $\mathscr{T}_{\Gamma}$ on $\mathfrak{A}$ generated by $\Gamma$ is also the locally convex topology generated by the directed set $\tilde{\Gamma}$ of all the $\mathscr{T}_{\Gamma}$-continuous $C^{*}$-semi-norms on $\mathfrak{A}$ so, for instance, we may define $\mathscr{A}(\mathfrak{H}, \Gamma)$ to be $\mathscr{A}(\mathfrak{A}, \tilde{\Gamma})$ etc. .... Notice that we have: $\tilde{\Gamma}=\{p \in \mathscr{N}(\mathfrak{Q})) \mid p \leqq \sup _{i \in I_{p}}\left(p_{i}\right)$ for a finite family $\left(p_{i}\right)_{i \in I_{p}}$ in $\left.\Gamma\right\}$. Many constructions introduced in Part I are only auxiliary constructions needed to state and to discuss the $m$-problem so it is natural to look for the dependence on $\Gamma$ of the $m(\Gamma)$-problem $[\Gamma$ being now an arbitrary subset of $\mathscr{N}(\mathfrak{H})]$; it is the object of this section.

Lemma 5. Let $x$ be an arbitrary element of $\mathfrak{A}$ and let $p_{1}$ and $p_{2}$ be two semi-norms on $\mathfrak{A}$ such that $p_{1}(\mathbb{1})=p_{2}(\mathbb{1})=1$ and $p_{i}\left(p_{i}(x) \mathbb{1}-x\right) \leqq p_{i}(x)$ for $i=1,2$. Then the semi-norm $p$ $=\sup \left(p_{1}, p_{2}\right)$ satisfies $\mathrm{p}(\mathbb{1})=1$ and $p(p(x) \mathbb{1}-x) \leqq p(x)$.

Proof. Suppose for instance that $p_{1}(x) \geqq p_{2}(x)$. Then, we have $\sup \left(p_{1}, p_{2}\right)$ $\left(\sup \left(p_{1}, p_{2}\right)(x) \mathbb{1}-x\right)=\sup _{i=1,2} p_{i}\left(p_{1}(x) \mathbb{1}-x\right)$. By assumption $p_{1}\left(p_{1}(x) \mathbb{1}-x\right) \leqq p_{1}(x)$ $=\sup \left(p_{1}, p_{2}\right)(x)$, and we have $p_{2}\left(p_{1}(x) \mathbb{1}-x\right) \leqq p_{2}\left(p_{2}(x) \mathbb{1}-x\right)+p_{2}\left(\left[p_{1}(x)-p_{2}(x)\right] \mathbb{1}\right)$ $=p_{1}(x)-\left(p_{2}(x)-p_{2}\left(p_{2}(x) \mathbb{1}-x\right)\right) \leqq p_{1}(x)$. So we have $p(p(x) \mathbb{1}-x) \leqq p(x)$ and, on the other hand $p(\mathbb{1})=1$ is obvious.

As usual, the positive cone $\mathfrak{A}^{+}$of $\mathfrak{A}$ is the convex cone in $\mathfrak{A}$ generated by $\{x * x \mid x \in \mathfrak{A}\}$.

Lemma 6. Let $h$ be an arbitrary hermitian element of $\mathfrak{A}$ and let $\Gamma$ be a set of $C^{*}$-seminorms on $\mathfrak{A}$. Then $h$ is in the $\mathscr{T}_{\Gamma^{-}}$-closure of $\mathfrak{H}^{+}$if and only if we have: $p(p(h) \mathbb{1}-h)$ $\leqq p(h), \forall p \in \Gamma$.

Proof. Let $\Gamma_{1}$ be the directed set of $C^{*}$-semi-norms on $\mathfrak{A}$ generated by $\Gamma$. We have $\mathscr{T}_{\Gamma_{1}}=\mathscr{T}_{\Gamma}$ and $\mathscr{A}\left(\mathfrak{H}, \Gamma_{1}\right)=\mathscr{A}(\mathfrak{A}, \Gamma)$. On the other hand, by the Lemma 5, we have:

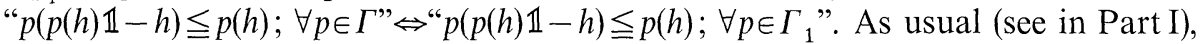
we may assume that $\mathscr{T}_{\Gamma}=\mathscr{T}_{\Gamma_{1}}$ is a Hausdorff topology on $\mathfrak{U}$ (otherwise replace $\mathfrak{A}$ by the factor algebra $\mathfrak{A} /\{0\}^{\mathscr{T}}$ ). So $h$ is in the $\mathscr{T}_{\Gamma}$-closure of $\mathfrak{U}^{+}$if and only if $h \in \mathfrak{A} \cap \mathscr{A}^{+}\left(\mathfrak{H}, \Gamma_{1}\right)$ and this is equivalent to $\pi_{p}(h)$ positive, $\forall p \in \Gamma_{1}$ [see Part I, Section 2, Lemma 3d)]. On the other hand, it is known that in a $C^{*}$-algebra with a unit, a hermitian element $x$ is positive if and only if it satisfies \|\|$x\|\mathbb{1}-x\| \leqq\|x\|$, [9]. Therefore $h=h^{*} \in \mathfrak{A}$ is in the $\mathscr{T}_{\Gamma^{-}}$-closure of $\mathfrak{A}^{+}$if and only if we have $p(p(h) \mathbb{1}-h)$ $\leqq p(h)$ for any $p \in \Gamma_{1}$ [i.e. $\left.\forall p \in \Gamma_{1},\|\| \pi_{p}(h)\left\|\mathbb{1}-\pi_{p}(h)\right\| \leqq\left\|\pi_{p}(h)\right\|\right]$ and, since this is equivalent to $p(p(h) \mathbb{1}-h) \leqq p(h)$ for any $p \in \Gamma$, this proves the Lemma 6 .

Lemma 7. Let $x$ be an arbitrary element of $\mathfrak{A}$. Then $p \mapsto p(p(x) \mathbb{1}-x)$ is a continuous mapping of $\mathscr{N}(\mathfrak{Q})$ in $\mathbb{R}$. 
Proof. Let $p_{0}$ be an arbitrary element of $\mathscr{N}(\mathfrak{U})$ and consider, for any $\varepsilon>0$, the neighborhood of $p_{0}$ in $\mathscr{N}(\mathfrak{U})$ defined by:

$$
\mathscr{V}_{p_{0}, \varepsilon}=\left\{p \in \mathscr{N}(\mathfrak{A}) \| p_{0}(x)-p(x)|\leqq \varepsilon / 2,| p_{0}\left(p_{0}(x) \mathbb{1}-x\right)-p\left(p_{0}(x) \mathbb{1}-x\right) \mid \leqq \varepsilon / 2\right\} .
$$

For any $p \in \mathscr{V}_{p_{0}, \varepsilon}$, we have: $\left|p_{0}\left(p_{0}(x) \mathbb{1}-x\right)-p(p(x) \mathbb{1}-x)\right| \leqq \mid p_{0}\left(p_{0}(x) \mathbb{1}-x\right)$ $-p\left(p_{0}(x) \mathbb{1}-x\right)|+| p\left(p_{0}(x) \mathbb{1}-x\right)-p(p(x) \mathbb{1}-x) \mid \leqq \varepsilon$, where we used $|p(a)-p(b)| \leqq p(a$ $-b)$ and $p(\mathbb{1})=1$. This prove that $p \mapsto p(p(x) \mathbb{1}-x)$ is continuous since $\varepsilon>0$ and $p_{0} \in \mathscr{N}(\mathfrak{U})$ are arbitrary.

Remark 3. Notice that $p \mapsto p(p(x) \mathbb{1}-x)$ is not uniformly continuous.

It follows from the last lemma and from the Lemma 5 that the set $\Gamma_{x}$ $=\{p \in \mathscr{N}(\mathfrak{U}) \mid p(p(x) \mathbb{1}-x) \leqq p(x)\}$ is a closed directed subset of $\mathscr{N}(\mathfrak{U})$ for any $x \in \mathfrak{U}$.

Proposition 1. Let $\Gamma$ be a set of $C^{*}$-semi-norms on $\mathfrak{A}$ and let $\overline{\mathfrak{A}^{+} \mathscr{T}_{r}}$ denote the closure of $\mathfrak{U}^{+}$in $\mathfrak{A}$ equipped with the locally convex topology $\mathscr{T}_{\Gamma}$ generated by $\Gamma$. Then the set $\left.\hat{\Gamma}=\{p \in \mathcal{N}(\mathfrak{U})) \mid p(p(h) \mathbb{1}-h) \leqq p(h), \forall h \in \overline{\mathfrak{U}}^{+} \mathscr{T}_{\Gamma}\right\}$ is the greatest set of $C^{*}$-semi-norms on $\mathfrak{U}$ for which we have: $\overline{\mathfrak{A}^{+} \mathscr{T} \hat{\Gamma}}=\overline{\mathfrak{A}}^{+} \mathscr{T}_{\Gamma} . \hat{\Gamma}$ is a closed directed subset of $\mathscr{N}(\mathfrak{A})$ and we have $\hat{\Gamma}=\tilde{\Gamma}$.

Proof. We have $: \hat{\Gamma}=\bigcap_{h \in \mathfrak{\mathscr { R }}^{+} \mathscr{T}_{\Gamma}} \Gamma_{h}$. So $\hat{\Gamma}$ is a closed directed subset of $\mathscr{N}(\mathfrak{A})$. The rest of the proof follows from the Lemma 6.

We know (Part I, Section 6, Proposition 2) that, if $\Gamma_{1}$ and $\Gamma_{2}$ are two sets of $C^{*}$ semi-norms on $\mathfrak{U}$ such that $\mathscr{T}_{\Gamma_{1}}$ is finer than $\mathscr{T}_{\Gamma_{2}}$, the canonical continuous *homomorphism $\pi_{\Gamma_{2} \Gamma_{1}}: \mathscr{A}\left(\mathfrak{U}, \Gamma_{1}\right) \rightarrow \mathscr{A}\left(\mathfrak{U}, \Gamma_{2}\right)$ has a restriction to $\mathfrak{B}\left(\mathfrak{U}, \Gamma_{1}\right)$ which is a surjective *-homomorphism of the $C^{*}$-algebra $\mathfrak{B}\left(\mathfrak{A}, \Gamma_{1}\right)$ on the $C^{*}$-algebra $\mathfrak{B}\left(\mathfrak{U}, \Gamma_{2}\right) \quad\left(\pi_{\Gamma_{2} \Gamma_{1}}\left(\mathfrak{B}\left(\mathfrak{U}, \Gamma_{1}\right)\right)=\mathfrak{B}\left(\mathfrak{U}, \Gamma_{2}\right)\right)$. Let us simply denote by $\pi_{\Gamma}$ (instead of $\pi_{\Gamma}, \mathscr{N}_{(\mathfrak{l})}$ the canonical ${ }^{*}$-homomorphism of $\mathscr{A}(\mathfrak{U}, \mathscr{N}(\mathfrak{U}))$ in $\mathscr{A}(\mathfrak{U}, \Gamma) ; \forall \Gamma \subset \mathscr{N}(\mathfrak{U})$. Then, since $\pi_{\Gamma}(\mathfrak{B}(\mathfrak{U}))=\mathfrak{B}(\mathfrak{U}, \Gamma)\left(\right.$ remembering that the $C^{*}$-algebra associated with $\mathfrak{A}$, $\mathfrak{B}(\mathfrak{U})$, is defined by $\mathfrak{B}(\mathfrak{U}, \mathscr{N}(\mathfrak{U}))=\mathfrak{B}(\mathfrak{U}))$, it follows that $\mathfrak{B}(\mathfrak{A}, \Gamma)$ is *-isomorphic with the factor $C^{*}$-algebra $\mathfrak{B}(\mathfrak{U}) / \pi_{\Gamma}^{-1}(0) \cap \mathfrak{B}(\mathfrak{U})$. In the following we shall identify $\mathfrak{B}(\mathfrak{U}, \Gamma)$ and $\mathfrak{B}(\mathfrak{U}) / \pi_{\Gamma}^{-1}(0) \cap \mathfrak{B}(\mathfrak{U})$ under this isomorphism writing therefore:

$$
\mathfrak{B}\left(\mathfrak{A}, \Gamma_{1}\right)=\mathfrak{B}\left(\mathfrak{A}, \Gamma_{2}\right) \quad \text { if } \quad \pi_{\Gamma_{1}}^{-1}(0) \cap \mathfrak{B}(\mathfrak{U})=\pi_{\Gamma_{2}}^{-1}(0) \cap \mathfrak{B}(\mathfrak{U}) ;
$$

where

$$
\Gamma_{1} \subset \mathscr{N}(\mathfrak{U}) \quad \text { and } \quad \Gamma_{2} \subset \mathscr{N}(\mathfrak{U}) .
$$

Let $\Gamma$ be a set of $C^{*}$-semi-norms on $\mathfrak{U}$ and let $\gamma_{\Gamma}: \mathfrak{U} \rightarrow \mathscr{A}(\mathfrak{A}, \Gamma)$ be the canonical continuous *-homomorphism of $\mathfrak{A}$ equipped with $\mathscr{T}_{\Gamma}$ in the associated complete Hausdorff topological *-algebra $\mathscr{A}(\mathfrak{A}, \Gamma)$. In the Part I (Section 6), we have defined $f(h) \in \mathscr{A}(\mathfrak{U}, \Gamma)$ for any continuous functions $f$ on $\mathbb{R}$ and for any hermitian element $h$ of $\mathscr{A}(\mathfrak{U}, \Gamma)$; furthermore, if $\mathscr{C}(\mathbb{R})$ is the *-algebra of complex continuous functions on $\mathbb{R}$ equipped with the topology of compact convergence, $f \mapsto f(h)$ is a continuous *homomorphism of $\mathscr{C}(\mathbb{R})$ in $\mathscr{A}(\mathfrak{U}, \Gamma)$. It will be convenient in the following to denote the spectrum of $\gamma_{\Gamma}(x)$ in $\mathscr{A}(\mathfrak{A}, \Gamma)$ by $\operatorname{Sp}_{\Gamma}(x)$, for any $x \in \mathfrak{A} ; \overline{\operatorname{Sp}_{\Gamma}(x)}$ will denote its closure in $\mathbb{C}$ (in $\mathbb{R}$ if $x=x^{*}$ ). 
Theorem 2. Let $\Gamma_{1}$ and $\Gamma_{2}$ be two sets of $C^{*}$-semi-norms on $\mathfrak{A}$; the following conditions are equivalent:

a) the $\Gamma_{1}$-strongly positive linear forms on $\mathfrak{A}$ and the $\Gamma_{2}$-strongly positive linear forms on $\mathfrak{A}$ are identical,

b) the $\mathscr{T}_{\Gamma_{1}}$-continuous positive linear forms on $\mathfrak{U}$ are $\Gamma_{2}$-strongly positive and the $\mathscr{T}_{\Gamma_{2}}$-continuous positive linear forms on $\mathfrak{A}$ are $\Gamma_{1}$-strongly positive,

c) the $\mathscr{T}_{\Gamma_{1}}$-closure of $\mathfrak{U}^{+}$coincides with its $\mathscr{T}_{\Gamma_{2}}$-closure (in $\left.\mathfrak{A}\right)$,

d) $\hat{\Gamma}_{1}$ and $\hat{\Gamma}_{2}$ are identical (in $\mathscr{N}(\mathfrak{U})$ ),

e) $\mathfrak{B}\left(\mathfrak{U}, \Gamma_{1}\right)=\mathfrak{B}\left(\mathfrak{U}, \Gamma_{2}\right)$ (i.e. $\left.\pi_{\Gamma_{1}}^{-1}(0) \cap \mathfrak{B}(\mathfrak{U})=\pi_{\Gamma_{2}}^{-1}(0) \cap \mathfrak{B}(\mathfrak{A})\right)$,

f) $\overline{\mathrm{Sp}_{\Gamma_{1}}(h)}=\overline{\mathrm{Sp}_{\Gamma_{2}}(h)}$ for any hermitian element $h$ of $\mathfrak{U}$.

Furthermore, under these conditions, for any linear form $\phi$ on $\mathfrak{A}$, a positive linear form $\omega$ on $\mathfrak{B}\left(\mathfrak{U}, \Gamma_{1}\right)=\mathfrak{B}\left(\mathfrak{A}, \Gamma_{2}\right)$ is a solution of the $m\left(\Gamma_{1}\right)$-problem (resp. $\tilde{m}\left(\Gamma_{1}\right)$ problem) for $\phi$ if and only if it is a solution of the $m\left(\Gamma_{2}\right)$-problem (resp. $\tilde{m}\left(\Gamma_{2}\right)$ problem) for $\phi$.

Proof. a $\Rightarrow$ b) since, for any $\Gamma \subset \mathscr{N}(\mathfrak{A})$, a $\mathscr{T}_{\Gamma}$-continuous positive linear form on $\mathfrak{U}$ is positive on the $\mathscr{T}_{\Gamma}$-closure of $\mathfrak{U}^{+}$which means that it is $\Gamma$-strongly positive.

b) $\Rightarrow$ c) since $\mathscr{T}_{\Gamma_{1}}$ and $\mathscr{T}_{\Gamma_{2}}$ are locally convex topology on $\mathfrak{U}$ and since $\mathfrak{U}^{+}$is a convex subset of $\mathfrak{A}\left[\overline{\mathfrak{U}}{ }^{+\mathscr{T}_{\Gamma}}\right.$ is the polar of the set of all the $\mathscr{T}_{\Gamma}$-continuous positive linear form and the set of all the $\Gamma$-strongly positive linear forms is the polar of $\overline{\mathfrak{U}}^{+\mathscr{T}_{\Gamma}}$ in the algebraic dual of $\left.\mathfrak{U} ; \forall \Gamma \subset \mathcal{N}(\mathfrak{U})\right][10]$.

c) $\Rightarrow$ a) by the very definition of strong positivity;

c) $\Leftrightarrow$ d) is trivial (see the Lemma 6);

d) $\Rightarrow \mathrm{e})$ is equivalent with $\mathfrak{B}(\mathfrak{U}, \hat{\Gamma})=B(\mathfrak{A}, \Gamma) ; \forall \Gamma \subset \mathscr{N}(\mathfrak{U})$.

Let us prove this equality which is equivalent to $\pi_{\Gamma \hat{\Gamma}}^{-1}(0) \cap \mathfrak{B}(\mathfrak{U}, \hat{\Gamma})=\{0\}$; $\forall \Gamma \subset \mathscr{N}(\mathfrak{U})$. Any continuous linear form on $\mathscr{A}(\mathfrak{U}, \hat{\Gamma})$ is a finite combination of positive continuous linear forms on $\mathscr{A}(\mathfrak{U}, \hat{\Gamma})$ and, on the other hand, $\mathfrak{B}(\mathfrak{U}, \hat{\Gamma}) \subset \mathscr{A}(\mathfrak{H}, \hat{\Gamma})$ implies that the continuous linear forms on $\mathscr{A}(\mathfrak{U}, \hat{\Gamma})$ (their restrictions to $\mathfrak{B}(\mathfrak{U}, \hat{\Gamma}))$ separates the points of $\mathfrak{B}(\mathfrak{H}, \hat{\Gamma})[\mathscr{A}(\mathfrak{U}, \hat{\Gamma})$ is a Hausdorff locally convex space]. It follows that, in order to prove our statement, it is sufficient to show that for any continuous positive linear form $\psi$ on $\mathscr{A}(\mathfrak{U}, \hat{\Gamma})$ there is a positive linear form $\omega$ on $\mathfrak{B}(\mathfrak{H}, \Gamma)$ for which we have: $\psi(x)=\omega\left(\pi_{\Gamma \hat{\Gamma}}(x)\right), \forall x \in \mathfrak{B}(\mathfrak{H}, \hat{\Gamma})$.

But let $\phi$ be the defined by $\phi(y)=\psi\left(\gamma_{\hat{\Gamma}}(y)\right), \forall y \in \mathfrak{A} ; \phi$ is $\Gamma$-strongly positive [by d) $\Leftrightarrow \mathrm{b})]$ since it is $\mathscr{T}_{\Gamma}$-continuous. So there is a solution $\omega$ of the $m(\Gamma)$-problem for $\phi$ and, since the representation of $\mathfrak{U}$ associated with $\phi$ is bounded, $\omega$ is unique (by the Theorem 1, for instance) and we have: $\psi\left(f\left(\gamma_{\hat{\Gamma}}(h)\right)=\omega\left(f\left(\gamma_{\Gamma}(h)\right)\right), \forall f \in \mathscr{C}_{(0)}(\mathbb{R})\right.$ and $\forall h \in \mathfrak{U}^{\hbar}$. This implies $\psi(x)=\omega\left(\pi_{\Gamma \hat{\Gamma}}(x)\right), \forall x \in \mathfrak{B}(\mathfrak{H}, \hat{\Gamma})$ [indeed $f\left(\gamma_{\Gamma}(h)\right)=\pi_{\Gamma \hat{\Gamma}}\left(f\left(\gamma_{\hat{\Gamma}}(h)\right)\right)$ and $\mathfrak{B}(\mathfrak{H}, \Gamma)$ is generated by the $\left.f\left(\gamma_{\Gamma}(h)\right), f \in \mathscr{C}_{(0)}(\mathbb{R}), h=h^{*} \in \mathfrak{U}\right]$.

e) $\Rightarrow \mathrm{f}$ ) because if e) is satisfied then $f\left(\gamma_{\Gamma_{1}}(h)\right)=0$ is equivalent to $f\left(\gamma_{\Gamma_{2}}(h)\right)=0$, $\forall h=h^{*} \in \mathfrak{U}$ and $\forall f \in \mathscr{C}_{(0)}(\mathbb{R})$; so the greatest closed subset $S_{h} \subset \mathbb{R}$ such that $f \in \mathscr{C}_{(0)}(\mathbb{R})$ and $f\left(S_{h}\right)=0$ imply $f\left(\gamma_{\Gamma_{1}}(h)\right)=0$ is also the greatest closed subset of $\mathbb{R}$ such that $f \in \mathscr{C}_{(0)}(\mathbb{R})$ and $f\left(S_{h}\right)=0$ imply $f\left(\gamma_{\Gamma_{2}}(h)\right)=0$. This means $\overline{\operatorname{Sp}_{\Gamma_{1}}(h)}=\overline{\operatorname{Sp}_{\Gamma_{2}}(h)}=S_{h}$ because we have: $\mathfrak{B}(\mathfrak{A}, \Gamma) \subset \prod_{p \in \Gamma} \mathfrak{B}_{p}(\mathfrak{A})$ and $\pi_{p}(\mathfrak{B}(\mathfrak{A}, \Gamma))$ $=\mathfrak{B}_{p}(\mathfrak{U})\left(\right.$ see in Part I) and $\operatorname{Sp}_{\Gamma}(h)=\bigcup_{p \in \Gamma} \operatorname{Sp}\left(\pi_{p}\left(\gamma_{\Gamma}(h)\right)\right.$ 

3).

f) $\Rightarrow$ c) because $h \in \overline{\mathfrak{A}^{+} \mathscr{T}_{\Gamma}}$ is equivalent to $\operatorname{Sp}_{\Gamma}(h) \subset \mathbb{R}^{+}$(see Part I, Section, Lemm

This achieves the proof of the equivalence of the conditions a) - $f$ ).

The conditions a), e), and f) clearly imply that any solution $\omega$ of the $\tilde{m}\left(\Gamma_{1}\right)$. problem for a linear form $\phi$ on $\mathfrak{A}$ is also a solution of the $\tilde{m}\left(\Gamma_{2}\right)$-problem anc conversely. It remains to show that the same it true for the $m$-problem. It is clearly sufficient to show that any solution $\omega$ of the $m(\hat{\Gamma})$-problem for a linear form $\phi$ on $\mathfrak{Q}$ is also a solution of the $m(\Gamma)$-problem for $\phi(\forall \Gamma \subset \mathcal{N}(\mathfrak{U}))$. The closures of $\{0\}$ in $\mathfrak{2}$ for $\mathscr{T}_{\hat{\Gamma}}$ and for $\mathscr{T}_{\Gamma}$ obviously coincide so we may suppose without restriction tha1 $\mathscr{T}_{\hat{\Gamma}}$ and $\mathscr{T}_{\Gamma}$ are Hausdorff topologies on $\mathfrak{A}$ (replace $\mathfrak{A}$ by $\mathfrak{A} /\{\overline{0}\}$ where $\{\overline{0}\}=\{\overline{0}\}^{\mathscr{T}_{\hat{I}}}$ $=\left\{\overline{\{0\}}^{\mathscr{T}}\right)$. Using the definition of the $m$-problem (Part I, Section 7, Definition $4^{\prime}$ ), we are reduced to show that $h=h^{*} \in \mathfrak{U}, b=b^{*} \in \mathfrak{B}(\mathfrak{U}, \Gamma)=\mathfrak{B}(\mathfrak{U}, \hat{\Gamma})$ and $h-b \in \mathscr{A}^{+}(\mathfrak{U}, \Gamma$ imply $h-b \in \mathscr{A}^{+}(\mathfrak{U}, \hat{\Gamma})$. However $h-b \in \mathscr{A}^{+}(\mathfrak{H}, \hat{\Gamma})$ is equivalent to $\psi(h-b) \geqq 0$ for all positive continuous linear form $\psi$ on $\mathscr{A}(\mathfrak{U}, \hat{\Gamma})$. But then, if $\psi$ is positive and $\mathscr{T}_{\hat{\Gamma}^{\text {. }}}$ continuous, we know that $\psi \uparrow \mathfrak{B}(\mathfrak{U}, \Gamma)(=\mathfrak{B}(\mathfrak{U}, \hat{\Gamma}))$ is the unique solution of the $m(\Gamma)$-problem for $\psi \uparrow \mathfrak{A}$ [by the same argument as in $\mathrm{d}) \Rightarrow \mathrm{e})]$. It follows that $\forall h \in \mathfrak{A}$ and $b=b^{*} \in \mathfrak{B}(\mathfrak{A}, \Gamma)$ such that $h-b \in \mathscr{A}^{+}(\mathfrak{A}, \Gamma)$ we have $\psi(h-b) \geqq 0$ for any continuous positive linear form $\psi$ on $\mathscr{A}(\mathfrak{H}, \hat{\Gamma})$ so we have $h-b \in \mathscr{A}^{+}(\mathfrak{H}, \hat{\Gamma})$.

Definition 1. Two sets $\Gamma_{1}$ and $\Gamma_{2}$ satisfying the equivalent conditions a) $-\mathrm{f}$ ) of the last theorem will be said to have the same support and we write $\operatorname{Supp}\left(\Gamma_{1}\right)=\operatorname{Supp}\left(\Gamma_{2}\right)$. This is obviously an equivalence relation on $\mathfrak{P}(\mathscr{N}(\mathfrak{A}))$ and the corresponding factor space will be called the set of supports. If $\Gamma_{1} \subset \hat{\Gamma}_{2} \subset \mathcal{N}$ ( $\mathfrak{Y}$ we say that the support of $\Gamma_{1}$ is contained in the support of $\Gamma_{2}$ and we write $\operatorname{Supp}\left(\Gamma_{1}\right) \subset \operatorname{Supp}\left(\Gamma_{2}\right)$ (this is an order relation).

Remark 4. Let $h$ be an arbitrary hermitian element of $\mathfrak{U}\left(h \in \mathfrak{U}^{h}\right)$ and let $\mathbb{C}[X]$ be the *-algebra of complex polynomials with respect to the indeterminate $X$. Then we may associate to any set $\Gamma$ of $C^{*}$-semi-norms on $\mathfrak{A}$ the set $\Gamma(h)$ of $C^{*}$-semi-norms on $\mathbb{C}[X]$ defined by:

$$
\Gamma(h)=\left\{p_{h} \mid p_{h}(P(X))=p(P(h)), \forall P(X) \in \mathbb{C}[X] ; p \in \Gamma\right\} .
$$

It is not hard to see [use condition $\mathrm{f}$ ) in the last theorem] that $\operatorname{Supp}\left(\Gamma_{1}\right)$ $=\operatorname{Supp}\left(\Gamma_{2}\right)$ is equivalent to $\operatorname{Supp}\left(\Gamma_{1}(h)\right)=\operatorname{Supp}\left(\Gamma_{2}(h)\right), \forall h \in \mathfrak{A}^{h}$, and that, for the *. algebras of polynomials, the above definition is consistant with the definition given in the Section 5 of Part I. It follows that the set of supports may be identified with a set of closed subsets of $\mathbb{R}^{22^{h}}\left[\operatorname{Supp}\left(\Gamma_{1}\right) \subset \operatorname{Supp}\left(\Gamma_{2}\right)\right.$ if and only if the corresponding inclusion holds in $\mathbb{R}^{\mathfrak{Q}^{h}}$ ].

\section{Homomorphisms and Tensor Algebras}

We already pointed out in Part I that the tensor algebras over involutive vector spaces are of particular interest since every *-algebra with unit is (in a non unique way however) the quotient of such a tensor algebra by a *-invariant two-sided ideal. An immediate consequence of the last theorem and of the Theorem 2 of Part I (Section 4) is the following proposition. 
Proposition 2. Let $E$ be an involutive vector space, let $E^{\prime}$ be $a^{*}$-invariant subspace of the dual space of $E$ which separates the points of $E$ and let $\Gamma_{E^{\prime}}$ be as in the Theorem 2 of Part I. Then $\hat{\Gamma}_{E^{\prime}}$ is the set of all the $C^{*}$-semi-norms on the tensor algebra $T(E)$.

So we have $\mathfrak{B}(T(E))=\mathfrak{B}\left(T(E), \Gamma_{E^{\prime}}\right)$ etc....

Generally when one considers a *algebra with unit as a factor space of some tensor algebra over an involutive vector space, this means that one is interested on a real vector space of hermitian elements in this algebra which is generating. This is, for instance, typically the case in quantum field theory when one considers the field operator smeared with real test functions. This suggests to generalize the definitions of the quasi-localizable $C^{*}$-algebra (Definition 6, Part I, Section 9) by the following one.

Definition 2. Let $E$ be a real vector space and let $T(E+i E)$ be the tensor algebra over the complexified vector space $E+i E$ of $E$ equipped with its canonical structure of *algebra with unit. We define the $C^{*}$-algebra associated with $E, \mathfrak{B}_{0}(E)$, to be the $C^{*}$ subalgebra of $\mathfrak{B}(T(E+i E))$ generated by $\left\{f(h) \in \mathfrak{B}(T(E+i E)) \mid h \in E\right.$ and $\left.f \in \mathscr{C}_{(0)}(\mathbb{R})\right\}$.

It should be clear from this definition and from the Proposition 2 that the quasilocalizable $C^{*}$-algebra $\hat{\mathfrak{B}}(M)$ is the $C^{*}$-algebra $\mathfrak{B}_{0}(\mathscr{D}(M ; \mathbb{R}))$ associated with the space. $\mathscr{D}(M ; \mathbb{R})$ of the real $C^{\infty}$-function with compact supports on $M$.

In order to complete the discussion, we must describe the invariance of the $m$ problem under *homomorphisms.

Proposition 3. Let $\alpha_{12}: \mathfrak{U}_{2} \rightarrow \mathfrak{U}_{1}$ be a homomorphism of ${ }^{*}$-algebras with units $\left(\alpha_{12}(\mathbb{1})\right.$ $=\mathbb{1})$, let $\Gamma_{1}\left(\right.$ resp. $\left.\Gamma_{2}\right)$ be a set of $C^{*}$-semi-norms on $\mathfrak{A}_{1}\left(\right.$ resp. $\left.\mathfrak{U}_{2}\right)$ and let us assume that $p \in \Gamma_{1}$ implies $p \circ \alpha_{12} \in \hat{\Gamma}_{2}\left(i . e . \alpha_{12}\right.$ is continuous from $\left(\mathfrak{U}_{2}, \mathscr{T}_{\hat{\Gamma}_{2}}\right)$ in $\left.\left(\mathfrak{U}_{1}, \mathscr{T}_{\Gamma_{1}}\right)\right)$. Then there is a unique *-homomorphism $\beta_{\alpha_{12}}: \mathfrak{B}\left(\mathfrak{H}_{2}, \Gamma_{2}\right) \rightarrow \mathfrak{B}\left(\mathfrak{H}_{1}, \Gamma_{1}\right)$ such that we have:

$$
\beta_{\alpha_{12}}(f(h))=f\left(\alpha_{12}(h)\right)^{4}, \forall h \in \mathfrak{U}_{2}^{h} \text { and } \forall f \in \mathscr{C}_{(0)}(\mathbb{R}) .
$$

If $\phi$ is a $\Gamma_{1}$-strongly positive linear form on $\mathfrak{A}_{1}$ and if $\omega$ is a solution of the $m\left(\Gamma_{1}\right)$ problem for $\phi$, then $\phi \circ \alpha_{12}$ is a $\Gamma_{2}$-strongly positive linear form on $\mathfrak{U}_{2}$ and $\omega \circ \beta_{\alpha_{12}}$ is a solution of the $m\left(\Gamma_{2}\right)$-problem for $\phi \circ \alpha_{12}$.

If $\alpha_{23}: \mathfrak{U}_{3} \rightarrow \mathfrak{U}_{2}$ is another homomorphism of *-algebras with units and if $\Gamma_{3}$ is a set of $C^{*}$-semi-norms on $\mathfrak{U}_{3}$ such that, for any $p \in \Gamma_{2}, p \circ \alpha_{23}$ is in $\hat{\Gamma}_{3}$; then $\forall p \in \Gamma_{1}, p \circ$ $\alpha_{12}{ }^{\circ} \alpha_{23}$ is in $\hat{\Gamma}_{3}$ and we have:

$$
\beta_{\alpha_{12} \circ \alpha_{23}}=\beta_{\alpha_{12}} \circ \beta_{\alpha_{23}} \text {. }
$$

If $\alpha$ is the identity mapping of $\mathfrak{U}_{2}$ onto itself, $\beta_{\alpha}$ is the identity mapping of $\mathfrak{B}\left(\mathfrak{Q}_{2}, \Gamma_{2}\right)$ onto itself.

Proof. Let $\alpha_{12}: \mathfrak{U}_{2} \rightarrow \mathfrak{U}_{1}$ be as in the proposition. Then $\alpha_{12}$ is continuous if $\mathfrak{U}_{1}$ is equipped with $\mathscr{T}_{\Gamma_{1}}$ and if $\mathfrak{U}_{2}$ is equipped with $\mathscr{T}_{\hat{\Gamma}_{2}}$; therefore there is a unique continuous *-homomorphism $\hat{\alpha}_{12}: \mathscr{A}\left(\mathfrak{U}_{2}, \hat{\Gamma}_{2}\right) \rightarrow \mathscr{A}\left(\mathfrak{A}_{1}, \Gamma_{1}\right)$ for which $\hat{\alpha}_{12}\left(\gamma_{\hat{\Gamma}_{2}}(h)\right)$ $=\gamma_{\Gamma_{1}}\left(\alpha_{12}(h)\right)$, where $\gamma_{\hat{\Gamma}_{2}}: \mathfrak{U}_{2} \rightarrow \mathscr{A}\left(\mathfrak{U}_{2}, \hat{\Gamma}_{2}\right)$ and $\gamma_{\Gamma_{1}}: \mathfrak{U}_{1} \rightarrow \mathscr{A}\left(\mathfrak{U}_{1}, \Gamma_{1}\right)$ are the canonical mappings and where $h$ runs over the set $\mathfrak{U}_{2}^{h}$ of all the hermitian elements of $\mathfrak{A}_{2}$.

4 Here we use the notation $f(h)$ to denote the element $f\left(\gamma_{\Gamma_{2}}(h)\right)$ of $\mathfrak{B}\left(\mathfrak{U}_{2}, \Gamma_{2}\right)$ where $h=h^{*} \in \mathfrak{A}_{2}$, $f \in \mathscr{C}_{(0)}(\mathbb{R})$ and $\gamma_{\Gamma_{2}}$ is the canonical mapping of $\mathfrak{U}_{2}$ in $\mathscr{A}\left(\mathfrak{P}_{2}, \Gamma_{2}\right)$. (The same convention is applied for $\left.\mathfrak{A}_{1}, \Gamma_{1}\right)$ 
Then, the restriction $\hat{\alpha}_{12} \uparrow \mathfrak{B}_{\infty}\left(\mathfrak{U}_{2}, \hat{\Gamma}_{2}\right)$ is easily seen to be a *homomorphism of $\mathfrak{B}_{\infty}\left(\mathfrak{U}_{2}, \hat{\Gamma}_{2}\right)$ in $\mathfrak{B}_{\infty}\left(\mathfrak{A}_{1}, \Gamma_{1}\right)$ for which we have $\hat{\alpha}_{12}\left(f\left(\gamma_{\hat{\Gamma}_{2}}(h)\right)\right)=f\left(\gamma_{\Gamma_{1}}\left(\alpha_{12}(h)\right)\right)$ for any $h \in \mathfrak{U}_{2}^{h}$ and $f \in \mathscr{C}_{(0)}(\mathbb{R})$. It follows that the restriction $\beta_{\alpha_{12}}$ of $\hat{\alpha}_{12}$ to $\mathfrak{B}\left(\mathfrak{A}_{2}, \hat{\Gamma}_{2}\right)$ is a *. homomorphism of $\mathfrak{B}\left(\mathfrak{U}_{2}, \hat{\Gamma}_{2}\right)$ in $\mathfrak{B}\left(\mathfrak{U}_{1}, \Gamma_{1}\right)$ and we have canonically: $\mathfrak{B}\left(\mathfrak{U}_{2}, \Gamma_{2}\right)$ $=\mathfrak{B}\left(\mathfrak{U}_{2}, \hat{\Gamma}_{2}\right)$ and $f\left(\gamma_{\Gamma_{2}}(h)\right)=f\left(\gamma_{\hat{\Gamma}_{2}}(h)\right)(=f(h)$ with our conventions $)$ for any $h \in \mathfrak{U}_{2}^{h}$ and $f \in \mathscr{C}_{(0)}(\mathbb{R})$. So $\beta_{\alpha_{12}}$ satisfies the condition of the proposition and is clearly unique under this condition since $\mathfrak{B}\left(\mathfrak{U}_{2}, \Gamma_{2}\right)$ is generated by $\left\{f(h) \in \mathfrak{B}\left(\mathfrak{U}_{2}, \Gamma_{2}\right) \mid h=h^{*} \in \mathfrak{U}_{2}\right.$ and $\left.f \in \mathscr{C}_{(0)}(\mathbb{R})\right\}$ (as $C^{*}$-algebra). The rest of the proof of this proposition is completely straightforward (use the Theorem 2).

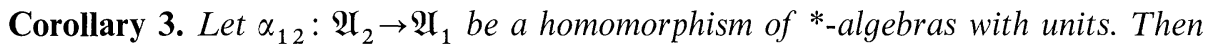
there is a unique *-homomorphism $\mathfrak{B}\left(\alpha_{12}\right): \mathfrak{B}\left(\mathfrak{U}_{2}\right) \rightarrow \mathfrak{B}\left(\mathfrak{U}_{1}\right)$ for which we have: $\mathfrak{B}\left(\alpha_{12}\right)(f(h))=f\left(\alpha_{12}(h)\right), \forall h=h^{*} \in \mathfrak{A}_{2}$ and $f \in \mathscr{C}_{(0)}(\mathbb{R})$. If $I_{\mathfrak{A}}$ is the identity mapping of the *-algebra with unit $\mathfrak{U}$ onto itself, $\mathfrak{B}\left(I_{\mathfrak{H}}\right)=I_{\mathfrak{B}(\mathfrak{a r})}$. If $\alpha_{23}: \mathfrak{U}_{3} \rightarrow \mathfrak{U}_{2}$ is another homomorphism of ${ }^{*}$-algebras with units, then we have: $\mathfrak{B}\left(\alpha_{12}\right) \circ \mathfrak{B}\left(\alpha_{23}\right)=\mathfrak{B}\left(\alpha_{12} \circ \alpha_{23}\right)$.

Proposition 4. Let $u: E \rightarrow F$ be a real-linear mapping of the real vector space $E$ in the real vector space $F$. Then, there is a unique *-homomorphism $\mathfrak{B}_{0}(u): \mathfrak{B}_{0}(E) \rightarrow \mathfrak{B}_{0}(F)$ for which we have: $\mathfrak{B}_{0}(u)(f(e))=f(u(e)), \forall e \in E$ and $\forall f \in \mathscr{C}_{(0)}(\mathbb{R})$. If $v: F \rightarrow G$ is another real-linear mapping, we have: $\mathfrak{B}_{0}(v \circ u)=\mathfrak{B}_{0}(v) \circ \mathfrak{B}_{0}(u)$. Furthermore we have: $\mathfrak{B}_{0}\left(I_{E}\right)=I_{\mathfrak{B}_{0}(E)}$.

Proof. Again the uniqueness of $\mathfrak{B}_{0}(u)$ immediately follows from the definition of $\mathfrak{B}_{0}(E)$ (Definition 2 above).

Let $\tilde{u}: T(E+i E) \rightarrow T(F+i F)$ be the unique homomorphism of *-algebras with units which extends $u$ (with obvious identifications). Then it is easy to see that the restriction $\mathfrak{B}_{0}(u)=\mathfrak{B}(\tilde{u})\left\lceil\mathfrak{B}_{0}(E)\right.$ of $\mathfrak{B}(\tilde{u}): \mathfrak{B}(T(E+i E)) \rightarrow \mathfrak{B}(T(F+i F))$ mapps the $C^{*}$-subalgebra $\mathfrak{B}_{0}(E)$ of $\mathfrak{B}(T(E+i E))$ in the $C^{*}$-subalgebra $\mathfrak{B}_{0}(F)$ of $\mathfrak{B}(T(F+i F))$ and satisfies the condition of the Proposition 4. The rest of the proof is immediate.

Remark 5. a) The Proposition 4 implies, in particular that there is a canonical group homomorphism of the group $\operatorname{Aut}(E)$ of all the real-linear inversible mapping of $E$ on itself in the group $\operatorname{Aut}\left(\mathfrak{B}_{0}(E)\right)$ of all the *-automorphisms of $\mathfrak{B}_{0}(E)$. This must be compared with the Proposition 5 of Part I.

b) Corollary 3 (resp. Proposition 4) implies that $\mathfrak{B}$ (resp. $\mathfrak{B}_{0}$ ) is a covariant functor of the category of *-algebras with units (resp. of the real vector spaces) in the category of $C^{*}$-algebras.

c) It would be of some interest to be able to define $\mathfrak{B}(\mathfrak{A})$ and $\mathfrak{B}_{0}(E)$ as solutions of universal problems (this could bring some light, for instance, on the connexion of the $m$-problem with the $\tilde{m}$-problem).

\section{Application to Essentially Self-adjoint Quantum Fields}

It will be convenient in this section to call hermitian scalar field a linear mapping $A$ of the real vector space $\mathscr{D}(M, \mathbb{R})$ of the real $C^{\infty}$-functions with compact supports on the Minkowski space $M=\mathbb{R}^{s+1}$ in the real vector space of the symmetric operators 
on a dense domain $D$ in a Hilbert space $\mathfrak{S}$ such that the following conditions are satisfied:

a) $A(h) D \subset D, \forall h \in \mathscr{D}(M, \mathbb{R})$,

b) there is a unit vector $\Omega \in D$ such that $D$ is the linear hull of $A\left(h_{1}\right) \ldots A\left(h_{N}\right) \Omega$ when $\left(h_{i}\right)$ runs over the finite families in $\mathscr{D}(M, \mathbb{R})$.

Let $A$ be a hermitian scalar field in the above sense, then of course $\sum \lambda_{i_{1} \ldots i_{n}} h_{i_{1}} \otimes \ldots \otimes h_{i_{n}} \mapsto \sum \lambda_{i_{1} \ldots i_{n}} A\left(h_{i_{1}}\right) \ldots A\left(h_{i_{n}}\right)$ defines a *-representation of the tensor algebra $T(\mathscr{D}(M))$ over the space $\mathscr{D}(M)$ of complex $C^{\infty}$-functions with compact supports on $M$. This *-representation is a cyclic representation with cyclic vector $\Omega$ and if $\phi_{\Omega}$ denote the vector state on $T(\mathscr{D}(M))$ corresponding to $\Omega$, we have canonically:

$$
\mathfrak{H}=\mathfrak{H}_{\phi_{\Omega}}, D=D_{\phi_{\Omega}}, \Omega=\Omega_{\phi_{\Omega}} \text { and } A(h)=\pi_{\phi_{\Omega}}(h), \forall h \in \mathscr{D}(M, \mathbb{R}) .
$$

Theorem 3. Let $A$ be hermitian scalar field and let $\phi_{\Omega}$ be the corresponding state on $T(\mathscr{D}(M))$ (as above). Suppose that for any real test function $h \in \mathscr{D}(M)$ the field operator $A(h)$ is essentially self-adjoint. Then, the $m$-problem for $\phi_{\Omega}$ is determined on the quasi-localizable $C^{*}$-algebra $\hat{\mathfrak{B}}(M)$ and we have: $\tilde{\omega}\left(f_{1}\left(h_{1}\right) \ldots f_{n}\left(h_{n}\right)\right)$

$=\left(\Omega \mid f_{1}\left(\overline{A\left(h_{1}\right)}\right) \ldots f_{n}\left(\overline{A\left(h_{n}\right)}\right) \Omega\right), \forall n \geqq 0, \forall f_{1}, \ldots, f_{n} \in \mathscr{C}_{(0)}(\mathbb{R}), \forall h_{1}, \ldots, h_{n} \in \mathscr{D}(M, \mathbb{R})$ and for any solution $\tilde{\omega}$ of the m-problem for $\phi_{\Omega}$. Let $\omega$ be the unique positive linear form on $\mathfrak{B}(M)$ obtained by restriction of an arbitrary solution $\tilde{\omega}$ of the m-problem for $\phi_{\Omega}$ and let $\left(\pi_{\omega}, \mathfrak{H}_{\omega}, \Omega_{\omega}\right)$ be the corresponding G.N.S. triplet. Then, we have canonically: $\mathfrak{H}_{\omega}=\mathfrak{H}, \quad \Omega_{\omega}=\Omega \quad$ and $\pi_{\omega}(f(h))=f(\overline{A(h)}) ; \forall f \in \mathscr{C}_{(0)}(\mathbb{R})$ and $\forall h \in \mathscr{D}(M, \mathbb{R}) \quad$ (where $\overline{A(h)}=A(h)^{*}$ is the closure of $\left.A(h)\right)$.

So $\pi_{\omega}(\hat{\mathfrak{B}}(M))$ generates the bounded observables of the field.

This theorem has not to be proved since it is a specific case of the Theorem 1 (supplemented by the fact that by the Proposition 2 and the Definition 2, the $C^{*}$ algebra $\hat{\mathfrak{B}}(M)$ is identical with $\mathfrak{B}_{0}(\mathscr{D}(M, \mathbb{R}))$.

Remark 6. a) The Part b) of the Theorem 6 in Part I follows from the Part a) of that theorem and from the above Theorem 3. However, even if $A$ is a nice local Wightman field which is essentially self-adjoint on its usual domain, we do not know in general if its spectral projections generate local rings. In other words, the corresponding representation of the localizable algebra may fail to be local.

b) In the above definition of a hermitian scalar field, the field may be not local and since translation invariance does not enter, it may happen that $\Omega$ has not the meaning of a vacuum.

c) Notice also that no continuity with respect to the test functions is assumed.

Lemma 8. Let $(A, \mathfrak{H}, \Omega)$ be a hermitian scalar field and let $\left(A_{\alpha}, \mathfrak{H}_{\alpha}, \Omega_{\alpha}\right)_{\alpha \in I}$ be a net of hermitian scalar fields such that we have: $\lim \left(\Omega_{\alpha} \mid A_{\alpha}\left(h_{1}\right) \ldots A_{\alpha}\left(h_{n}\right) \Omega_{\alpha}\right)$ $=\left(\Omega \mid A\left(h_{1}\right) \ldots A\left(h_{n}\right) \Omega\right)$ for any finite family $h_{1}, \ldots, h_{n}$ in $\mathscr{D}(M)$. Let $\omega_{\alpha}$ be, for each $\alpha \in I$, the restriction to $\hat{\mathfrak{B}}(M)$ of an arbitrary solution of the $m$-problem for $\phi_{\Omega_{\alpha}}$. Then there is a weakly convergent subnet of $\left(\omega_{\alpha}\right)$ and the limit $\omega$ of such a subnet is the restriction to $\hat{\mathfrak{B}}(M)$ of a solution of the $m$-problem for $\phi_{\Omega}$. If the $m$-problem for $\phi_{\Omega}$ is determined on $\hat{\mathfrak{B}}(M)$, then $\left(\omega_{\alpha}\right)$ is weakly convergent. 
Proof. Let $\left(\omega_{\alpha^{\prime}}\right)$ be a weakly convergent subnet of $\left(\omega_{\alpha}\right)$ and let $\omega$ be its weak limit [such $\left(\omega_{\alpha^{\prime}}\right)$ exist since the set of all the states on a $C^{*}$-algebra is weakly compact]. Let

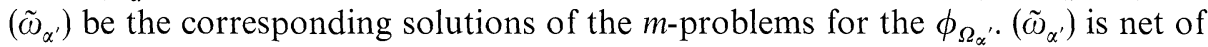
states on $\mathfrak{B}(T(\mathscr{D}(M))$ ) so (again by compacity) there is a weakly convergent subnet $\left(\tilde{\omega}_{\alpha^{\prime \prime}}\right)$ of $\left(\tilde{\omega}_{\alpha^{\prime}}\right)$. By the Lemma 4 , its limit $\tilde{\omega}$ is solution of the $m$-problem for $\phi_{\Omega}$ and, on the other hand, we clearly have $\omega=\tilde{\omega} \uparrow \hat{\mathfrak{B}}(M)$.

Remark 7. Roughly speaking, this means that one cannot miss the right result if one starts with an approximation $\left(A_{\alpha}, \mathfrak{H}_{\alpha}, \Omega_{\alpha}\right)$ then constructs $\omega_{\alpha}$ as above and obtains $\omega$ by compactness in the weak dual of the quasi-localizable $C^{*}$-algebra $\hat{\mathfrak{B}}(M)$. Indeed, $(A, \mathfrak{H}, \Omega)$ can be reconstructed from $\omega$ because by Corollary 2 we have $\mathfrak{H}=\mathfrak{H}_{\phi_{\Omega}} \subset \mathfrak{H}_{\omega}$, $\Omega=\Omega_{\omega}$, and by Lemmas 1 and 2 we have $A(h)=\pi_{\tilde{\omega}}(h) \uparrow \pi_{\tilde{\omega}}(T(\mathscr{D}(M))) \Omega$ where $\tilde{\omega}$ is any state on $\mathfrak{B}(T(\mathscr{D}(M)))$ extending $\omega$. $A$ does not depend on the choice of the extension $\tilde{\omega}$ of $\omega$.

\section{Example: A Class of Jaffe Cut-off Fields $[6]^{5}$}

We want to discuss the case of interacting cut-off hamiltonian field theory. In order to be explicit, we shall concentrate on $\lambda \phi^{4}$ theory and on a specific cut-off introduced by Jaffe in his thesis [6]; it must however be clear from the work of Jaffe that the arguments used here may be generalized to any polynomial interaction of degree $d \geqq 2$ which is bounded from below and to other types of cut-off which incorporate both an ultra-violet cut-off and a spatial cut-off.

We work in space-time $M=\mathbb{R}^{1+s}=\left\{(t, r) \mid t \in \mathbb{R}, \boldsymbol{r} \in \mathbb{R}^{s}\right\}$ and we shall deal with a cut-off which consists to replace the interaction picture time zero field $\phi(0, r)$ and its conjugate momentum $\pi(0, \boldsymbol{r})$ by two very regular objects on space $\left(\boldsymbol{r} \in \mathbb{R}^{s}\right) \phi_{N}(0, \boldsymbol{r})$ $=\phi_{N}(\boldsymbol{r})$ and $\pi_{N}(0, \boldsymbol{r})=\pi_{N}(\boldsymbol{r})$ which describe a system with $N$ degrees of freedom (for each integer $N \geqq 1$ ).

So let us consider in the Hilbert space $\mathfrak{Y}_{N}=L^{2}\left(\mathbb{R}^{N}\right)$ the $2 N$ operators $\left(p_{n}, q_{n}\right)$ defined on the dense domain $D_{N}^{1}=\mathscr{S}\left(\mathbb{R}^{N}\right)$ by:

$$
\Phi\left(q_{1}, \ldots, q_{n}\right) \mapsto-i \frac{\partial \Phi}{\partial q_{n}}\left(q_{1}, \ldots, q_{N}\right) \quad \text { and } \quad \Phi\left(q_{1}, \ldots, q_{N}\right) \mapsto q_{n} \Phi\left(q_{1}, \ldots, q_{N}\right),
$$

$\Phi \in D_{N}^{1}$. As it is well known, $D_{N}^{1}$ is a dense stable domain for these operators on which they are essentially self-adjoint.

Let $\left(e_{n}\right)_{n \in \mathbb{N}}$ denote a fixed orthonormal basis of the Hilbert space $L^{2}\left(\mathbb{R}^{s}\right)$ which consists of real valued function in $\mathscr{S}\left(\mathbb{R}^{s}\right)$ (for instance the Hermite functions). For each positive integer $N$, we define $\phi_{N}$ and $\pi_{N}$ by:

$$
\phi_{N}(\boldsymbol{r})=\phi_{N}(0, \boldsymbol{r})=\sum_{n=1}^{n=N} e_{n}(\boldsymbol{r}) q_{n} ; \quad \pi_{N}(\boldsymbol{r})=\pi_{N}(0, \boldsymbol{r})=\sum_{n=1}^{n=N} e_{n}(\boldsymbol{r}) p_{n} .
$$

For any real tempered distribution $T \in \mathscr{S}\left(\mathbb{R}^{s}\right)^{\prime},[11]$, the operators

$$
\left\langle T, \phi_{N}\right\rangle=\sum_{n \leqq N}\left\langle T, e_{n}\right\rangle q_{n} \text { and }\left\langle T, \pi_{N}\right\rangle=\sum_{n \leqq N}\left\langle T, e_{n}\right\rangle p_{n}
$$

are essentially self-adjoint on $D_{N}^{1}$ and map $D_{N}^{1}$ into itself.

5 Jaffe suggested to us several simplifications for the content of this section. The author thanks him for these suggestions 
The sense in which $\left(\phi_{N}, \pi_{N}\right)$ is a cut-off versus for the canonical pair $(\phi, \pi)$ must be clear; so for a formal interaction picture hamiltonian, we define the corresponding cut-off hamiltonian to be the operator in $\mathfrak{S}_{N}=L^{2}\left(\mathbb{R}^{N}\right)$ obtained by substitution of $\left(\phi_{N}, \pi_{N}\right)$ to $(\phi, \pi)$ in the corresponding formal expression. We shall concentrate on $\lambda \phi^{4}$ theory and, since from perturbation theory for $s=3$ we expect some mass and coupling constant renormalizations, we shall allow a dependence on $N$ for these parameters. Therefore we define $H_{N}$ on $D_{N}^{1}$ by:

$$
H_{N}=\int d^{s} r\left\{\frac{1}{2}\left[\pi_{N}(\boldsymbol{r})^{2}+\left(\boldsymbol{\nabla} \phi_{N}(\boldsymbol{r})\right)^{2}+m^{2} \phi_{N}(\boldsymbol{r})^{2}\right]+\lambda_{N} \phi_{N}(\boldsymbol{r})^{4}-\frac{\delta m_{N}^{2}}{2} \phi_{N}(\boldsymbol{r})^{2}\right\}-E_{N} \cdot(3)
$$

Using the formula (2), we obtain: $\left(\Delta_{N}=\sum_{n=1}^{n=N}\left(\frac{\partial}{\partial q_{n}}\right)^{2}\right)$

$$
H_{N}=\frac{1}{2} \sum_{n=1}^{n=N} p_{n}^{2}+V_{N}\left(q_{1}, \ldots, q_{N}\right)=-\frac{1}{2} \Delta_{N}+V_{N}\left(q_{1}, \ldots, q_{N}\right)
$$

where

$$
\begin{aligned}
V_{N}\left(q_{1}, \ldots, q_{N}\right)= & \lambda_{N}\left[\int e_{n_{1}} e_{n_{2}} e_{n_{3}} e_{n_{4}}\right] q_{n_{1}} q_{n_{2}} q_{n_{3}} q_{n_{4}} \\
& +\frac{1}{2}\left[\int\left(\nabla e_{n}\right)\left(\nabla e_{m}\right)+\left(m^{2}-\delta m_{N}^{2}\right) \delta_{n m}\right] q_{n} q_{m}-E_{N} .
\end{aligned}
$$

$H_{N}$ is a symmetric operator ${ }^{6}$ on $D_{N}^{1}$ and $H_{N} D_{N}^{1} \subset D_{N}^{1}$; the closure of $\mathrm{H}_{N}$ will again be denoted by $H_{N}$. The "free part"

$$
H_{N}^{(0)}=\int d^{s} r\left\{\frac{1}{2}\left[\pi_{N}^{2}+\left(\nabla \phi_{N}\right)^{2}+m^{2} \phi_{N}^{2}\right]\right\} \quad \text { (its closure) }
$$

is a well-known operator which is a semi-bounded (from below) self-adjoint operator with a pure discrete spectrum and lowest eigenvalue of multiplicity one. We shall denote the corresponding normalized eigenvector by $\Omega_{N}^{(0)}$ and refer to it as the Fock vacuum. We have: $\Omega_{N}^{(0)} \in D_{N}^{1}=\mathscr{S}\left(\mathbb{R}^{N}\right)$. Let $\Delta E_{N}^{(0)}$ be the difference between the lowest eigenvalue distinct from those corresponding to $\Omega_{N}^{(0)}$ and this last one, we have:

$$
\Delta E_{N}^{(0)}>m \text { and } \quad \lim _{N \rightarrow \infty} \Delta E_{N}^{(0)}=m=\inf _{N}\left(\Delta E_{N}^{(0)}\right) .
$$

In what follows we shall suppose that either $\lambda_{N}>0$, or $\lambda_{N}=0$ and $\delta m_{M}=0$. Under this assumption $V\left(q_{1}, \ldots, q_{N}\right)$ is a polynomial of degree 4 or 2 in the $q_{n}$ which is bounded from below and we have the following result [6].

Jaffe's Theorem. $H_{N}$ is self-adjoint, bounded from below, has a pure discrete spectrum and its minimum eigenvalue has multiplicity one. The resolvant of $H_{N}$ is compact and the eigenfunctions of $H_{N}$ belong to $D_{N}^{1}=\mathscr{S}\left(\mathbb{R}^{N}\right)$ and form a total set in $\mathfrak{S}_{N}=L^{2}\left(\mathbb{R}^{N}\right)$. $D_{N}^{1}$ is stable by $e^{i t H_{N}}, \forall t \in \mathbb{R}\left(e^{i t H_{N}} D_{N}^{1} \subset D_{N}^{1}\right)$. Furthermore the cyclic subspace $D_{N}^{(0)}$ generated from $\Omega_{N}^{(0)}$ by the polynomials in $p_{n}, q_{m}$ is a core for $H_{N}$.

In what follows, we shall suppose that $\lambda_{N} \geqq 0, \delta m_{M}$, and $E_{N}$ are chosen in such a way that the minimum eigenvalue of $H_{N}$ is zero (the corresponding normalized

6 It is of course understood that the parameters $m, \lambda_{N}, \delta m_{N}$, and $E_{N}$ are real 
eigenvector, called the vacuum, will be denoted by $\Omega_{N}$ ) and that the next smallest eigenvalue $E_{1 N}$ is equal to $\Delta E_{N}^{(0)}$ :

$$
E_{1 N}=\Delta E_{N}^{(0)}>m>0\left(\lim _{N \rightarrow \infty} E_{1 N}=m\right) .
$$

Notice that this is a renormalization prescription for the mass of the theory and that it does not determine $\lambda_{N}$ (here we do not impose conditions for the coupling constant renormalization).

Now let us define $\phi_{N}(t, r)=\phi_{N}(x)$ on $D_{N}^{1}$ by:

$$
\begin{aligned}
& \text { - } \phi_{N}(t, \boldsymbol{r})=e^{i t H_{N}} \phi_{N}(0, \boldsymbol{r}) e^{-i t H_{N}} \text {, } \\
& \phi_{N}(h)=\int d t d^{s} r h(t, r) \phi_{N}(t, r) .
\end{aligned}
$$

In order to obtain self-adjointness for the space-time smeared field operator, we follow Glimm and Jaffe $[15,16]$ (see also [17]).

Lemma 9. There exist positive constants $a_{N}, b_{N}$, and $c_{N}$ for which we have:

$$
\begin{aligned}
-\left(\Phi \mid\left(H_{N}+c_{N} \mathbb{1}\right) \Phi\right) & \leqq\left(\Phi \mid \phi_{N}(t, \boldsymbol{r}) \Phi\right) \leqq\left(\Phi \mid\left(H_{N}+c_{N} \mathbb{1}\right) \Phi\right) \text { and } \\
\left\|\phi_{N}(t, \boldsymbol{r}) \Phi\right\| & \leqq\left\|\left(a_{N} H_{N}+b_{N} \mathbb{1}\right)^{1 / 2} \Phi\right\|, \quad \forall \Phi \in D_{N}^{1} \quad \text { and } \quad \forall(t, \boldsymbol{r}) \in M .
\end{aligned}
$$

Proof. From the definition (8) it follows that it is sufficient to prove this inequality for $t=0$, and from (2) it follows that it is sufficient to show that they hold when $\phi(t, \boldsymbol{r})$ is replaced by $q_{n}(1 \leqq n \leqq N)$. But then, the first follows from (4) and the fact that we have $\left|q_{n}\right| \leqq V_{N}\left(q_{1} \ldots q_{n}\right)+c_{N}^{\prime}$ for some positive $c_{N}^{\prime}$ and the second from the fact that we have $q_{n}^{2} \leqq V_{N}\left(q_{1}, \ldots, q_{N}\right)+b_{N}^{\prime}$ if $\lambda_{N} \neq 0$ and, if $\lambda_{N}=0, q_{n}^{2} \leqq \frac{2}{m^{2}} V_{N}\left(q_{1}, \ldots, q_{N}\right)$ $+b_{N}^{\prime}$ for some $b_{N}^{\prime}>0$.

Lemma 10. Let $h$ be an arbitrary real test function of $\mathscr{D}(M)$. Then $\overline{\phi_{N}(h)}$ is essentially self-adjoint on any core for $H_{N}$.

Proof. The second inequality of Lemma 9 implies that we have:

$$
\left\|\phi_{N}(h) \Phi\right\| \leqq\|h\|_{L^{1}}\left\|\left(a_{N} H_{N}+b_{N} \mathbb{1}\right)^{1 / 2} \Phi\right\|, \quad \forall \Phi \in D_{N}^{1} .
$$

It follows that the domain $\operatorname{dom}\left(\overline{\phi_{N}(h)}\right)$ of the closure $\overline{\phi_{N}(h)}$ of $\phi_{N}(h)$ contains the completion of $D_{N}^{1}$ for the norm $\Phi \mapsto\left(\left\|\left(H_{N}+\frac{b_{N}}{a_{N}} \mathbb{1}\right)^{1 / 2} \Phi\right\|^{2}+\|\Phi\|^{2}\right)^{1 / 2}$ which [in fact it is $\left.\operatorname{dom}\left(H_{N}{ }^{1 / 2}\right)\right]$ clearly contains the domain $\operatorname{dom}\left(H_{N}\right)$ of the positive self-adjoint operator $H_{N}$. So we have:

$$
\operatorname{dom}\left(\overline{\phi_{N}(h)}\right) \supset \operatorname{dom}\left(H_{N}\right),
$$

and the same argument as above shows that for any core $C$ for $H_{N}, \operatorname{dom}\left(\overline{\phi_{N}(h)} \uparrow C\right)$ contains $\operatorname{dom}\left(H_{N}\right)$ and therefore that we have $\left.\overline{\left(\phi_{N}(h)\right.} \uparrow C\right)=\overline{\phi_{N}(h)}$.

In order to achieve the proof of Lemma 10, it is sufficient to show that $\overline{\phi_{N}(h)}$ is self-adjoint. Let us first remark that the following identity holds on $D_{N}^{1}$

$$
\phi_{N}\left(h^{\prime}\right)=i\left[H_{N}, \phi_{N}(h)\right], \quad \text { with } \quad h^{\prime}(t, \boldsymbol{r})=\frac{\partial h}{\partial t}(t, \boldsymbol{r}) \quad(\in \mathscr{D}(M)) .
$$


It follows that we have:

$$
\pm i\left\{\left(H_{N} \Phi \mid \overline{\phi_{N}(h)} \Phi\right)-\left(\overline{\phi_{N}(h)} \Phi \mid H_{N} \Phi\right)\right\}= \pm\left(\Phi \mid \overline{\phi_{N}\left(h^{\prime}\right)} \Phi\right) \leqq\left\|h^{\prime}\right\|_{L^{1}}\left(\Phi \mid\left(H_{N}+c_{N} \mathbb{1}\right) \Phi\right),
$$

for any $\Phi \in D_{N}^{1}$ (by Lemma 9) and therefore for any $\Phi \in \operatorname{dom}\left(H_{N}\right)$ by the above argument. The self-adjointness of $\overline{\phi_{N}(h)}$ is then a consequence of the following theorem which may be found in the book of Faris [12] (Theorem 12-1, p. 79; see also in [17] the [Jaffe's] Theorem 2.2).

Theorem. Let $Q$ be a symmetric operator and $H$ a positive self-adjoint operator. Assume that

i) $\operatorname{dom}(H) \subset \operatorname{dom}(Q)$

and for some constant $c$ and all $\Phi$ in $\operatorname{dom}(H)$,

ii) $\pm i\{(H \Phi \mid Q \Phi)-(Q \Phi \mid H \Phi\} \leqq c(\Phi \mid H \Phi)$.

Then $Q$ is essentially self-adjoint.

So applying this theorem with $Q=\phi_{N}(h), H=H_{N}+c_{N} \mathbb{1}$ and $c=\left\|h^{\prime}\right\|_{L^{1}}$ we obtain the desired result.

This lemma implies in particular that $\phi(h)$ is essentially self-adjoint on $D_{N}^{(0)}$.

Let us define the domain $D_{N}$ to be the linear hull of the vectors $\phi_{N}\left(h_{1}\right) \ldots \phi_{N}\left(h_{N}\right) \Omega_{N}$ when $\left(h_{1}, \ldots, h_{n}\right)$ runs over the finite families in $\mathscr{D}(M)$. Clearly $D_{N} \subset D_{N}^{1}, H_{N} D_{N} \subset D_{N}, \exp \left(i t H_{N}\right) D_{N} \subset D_{N} ; \forall t \in \mathbb{R}$.

There is a choice of the phase such that $\Omega_{N}\left(q_{1}, \ldots q_{N}\right)$ is strictly positive, $\forall\left(q_{1}, \ldots q_{N}\right) \in \mathbb{R}^{N}$; furthermore, $\Omega_{N} \in \mathscr{S}\left(\mathbb{R}^{N}\right)=D_{N}^{1}$ decreases faster (strictly faster if $\left.\lambda_{N}>0\right)$ than a gaussian at infinity. It follows that $\Omega_{N}^{2} d^{N} q$ is the solution of a determined moment problem and therefore, the polynomials $P\left(q_{1}, \ldots q_{N}\right)$ are dense in $L^{2}\left(\mathbb{R}^{N}, \Omega_{N}^{2} d^{N} q\right)$ which implies that the functions $P\left(q_{1}, \ldots q_{N}\right) \Omega_{N}\left(q_{1}, \ldots, q_{N}\right)$ are dense in $\mathfrak{H}_{N}=L^{2}\left(\mathbb{R}^{N}\right)$. On the other hand, it is easily seen that the closure of $D_{N}$ in $\mathfrak{H}_{N}$ contains these functions; so $D_{N}$ is dense. Since $D_{N}$ is dense, contained in dom $\left(H_{N}\right)$ and invariant by $e^{i t H_{N}}$, we have the following lemma.

Lemma 11. $D_{N}$ is a core for $H_{N}$.

Lemma 11 implies that $\left(\phi_{N}, \mathfrak{S}_{N}, \Omega_{N}\right)$ is an essentially self-adjoint scalar field (with our conventions), so we may apply to it the Theorem 3 . But since we expect for $s=3$ some "wave function" renormalization, we shall allow a dependence on $N$ of the normalization of the field operator. So we define the field $A_{N}$ by:

$$
A_{N}(h)=Z_{N}^{-1 / 2}\left[\phi_{N}(h)-\left(\Omega_{N} \mid \phi_{N}(h) \Omega_{N}\right)\right],
$$

for $h \in \mathscr{D}(M)$ and where $\Omega_{N}$ is the ground state of $H_{N} ; Z_{N}$ being a strictly positive constant which may be fixed, for instance by the following procedure. Let $A^{(0)}$ be the usual free field of mass $m$ (hermitian scalar free field), let $f \in \mathscr{S}\left(\mathbb{R}^{s+1}\right)$ be such that $A_{N}(f) \Omega_{N} \neq 0$ and such that the support of its Fourier transform $\hat{f}$ interset the physical spectrum of $A^{(0)}$ only on the mass shell of $A^{(0)}$, then choose $Z_{N}$ in such a way that $\left\|A_{N}(f) \Omega_{N}\right\|=\left\|A^{(0)}(f) \Omega^{(0)}\right\|$, where $\Omega^{(0)}$ is the usual vacuum in the Fock space of the free field of mass $m\left(A_{N}(t, r)\right.$ being well defined on $D_{N}^{1}$ by $A_{N}(t, r)$ $\left.=Z_{N}^{-1 / 2}\left[\phi_{N}(t, \boldsymbol{r})-\left(\Omega_{N} \mid \phi_{N}(t, r) \Omega_{N}\right)\right]\right)$. In any case (i.e. for any choice of $\left.Z_{N}>0\right)$ we may summarize the situation by the following statement. 
Theorem 4. The cut-off field $A_{N}=\left(A_{N}, \mathfrak{S}_{N}, \Omega_{N}\right)$ satisfies the assumptions of Theorem 3 .

Therefore, the $m$-problem for $\phi_{\Omega_{N}}$ is determined on the quasi-localizable $C^{*}$-algebra $\hat{\mathfrak{B}}(M)$. Let $\pi_{N}$ be the corresponding unique representation of $\hat{\mathfrak{B}}(M)$ in $\mathfrak{S}_{N}$ for which $\pi_{N}(f(h))=f\left(\overline{A_{N}(h)}\right) \in \mathscr{L}\left(\mathfrak{H}_{N}\right), \forall h=h^{*} \in \mathscr{D}(M)$ and $\forall f \in \mathscr{C}_{(0)}(\mathbb{R})$; we define the state $\omega_{N}$ on $\mathfrak{B}(M)$ associated to the above theory by:

$$
\omega_{N}(x)=\left(\Omega_{N} \mid \pi_{N}(x) \Omega_{N}\right), \quad \forall x \in \hat{\mathfrak{B}}(M) .
$$

Clearly (by G.N.S. construction + Lemmas 1 and 2) the knowledge of $\omega_{N}$ is equivalent to the knowledge of the whole theory $\left(\mathfrak{H}_{N}, H_{N}, A_{N}\right.$ etc....). What has been gained in this translation is that the space of states on $\hat{\mathfrak{B}}(M)$ equipped as usual with the weak topology is compact (remembering that this topology is reasonably physically relevant [19]). So from the sequence $\omega_{N}$, one may extract a convergent subnet. Let $\subseteq$ be the set of the limits of these convergent subnet. The natural question is then the following one: Is there a choice of the sequence $\left(\lambda_{N}, Z_{N}\right)$ for which $\subseteq$ contains at least an interesting point? Clearly an interesting point would be a state $\omega$ on $\hat{\mathfrak{B}}(M)$ such that, if $\left(\pi_{\omega}, \mathfrak{H}_{\omega}, \Omega_{\omega}\right)$ is the associated G.N.S. triplet and $\mathscr{F}_{b}$ denote the set of the non-empty open bounded subsets of $M$, the family $\left(\pi_{\omega}(\mathfrak{B}(\mathcal{O}))^{\prime \prime}\right)_{\Theta_{\in} \mathscr{F}_{b}}{ }^{7}$ of von Neumann algebras satisfies the assumptions of the theory of Araki and Haag $[13,14]$ adapted to the situation where one kind of neutral scalar particle of mass $m$ is present with vacuum $\Omega_{\omega^{*}}$. Of course, for the trivial choice $\lambda_{N}=0$ [which implies $\delta m_{N}=0$ with our convention formula (7)] and $Z_{N}=1 \forall N$, the Wightman distributions of the cut-off fields converge to the Wightman distributions of the free scalar neutral field of mass $m$ which is an essentially self-adjoint hermitian scalar field; so, by Theorem 3 and Lemma $8, \omega_{N}$ converge weakly to the vacuum expectation values of the bounded observables of the free field. In the case $\lambda_{N}>0(\forall N)$, it will be very difficult (and this is out of the scope of this paper) to recover the locality and the Poincare invariance when one removes the cut-off. However, since the cut-off fields are time-translation invariant and satisfy the spectrum condition in the time direction with a fixed gap $m$ and a unique $\left(\Omega_{N}\right)$, one may expect that the same holds for the "limits" (in the above sense).

\section{Conclusion}

Suppose that $\omega$ is a state on $\hat{\mathfrak{B}}(M)$ which is obtained, by weak compactness argument, from states corresponding to cut-off field theories as in the previous section. If we try to interpret $\omega$ as a vacuum state of some "limit" theory, a first difficulty arises because the G.N.S. Hilbert space $\mathfrak{H}_{\omega}$ may not be separable.

In quantum field theory $[20,21]$, the separability of the Hilbert space is a consequence of the continuity properties of the corresponding *-representation of the tensor algebra over the space of the test functions (using either the separability or the nuclearity of this topological *-algebra). At this point, it is worth noticing that all the constructions and the results of this work are purely algebraic. Furthermore

$7 \mathfrak{B}(\mathcal{O})$ being defined as in Part I, Section $9 \mathrm{a}) ; \mathfrak{B}(\mathcal{O})$ is a $C^{*}$-subalgebra of $\hat{\mathfrak{B}}(M), \forall \mathcal{O} \in \mathscr{F}_{b}$ 
Theorem 2 and Proposition 2 (above) show that the continuous positive linear forms on the tensor algebra over the test functions which correspond to bounded representations already separate the quasi-localizable $C^{*}$-algebra. So continuity will not reduce this $C^{*}$-algebra, and at the " $C^{*}$-algebraic level" one must use a nonseparable $C^{*}$-algebra in order to deal with "sufficiently many" field theories. The only way to escape is to remark that since continuity selects a subspace of the algebraic dual of the space of test functions we may expect that correspondingly one can find solutions for the associated $m$-problems in a (separating) subspace of the topological dual space of the quasi-localizable $C^{*}$-algebra such that the quasilocalizable $C^{*}$-algebra be separable for the corresponding weak topology. It must be clear that similar considerations apply when we are interested in the $m$-problem for continuous strongly positive linear forms on locally convex *-algebras.

For instance if $E$ is some locally convex space with topological dual $E^{\prime}$ and if $\phi$ is a strongly positive linear form on the symmetric tensor algebra $S(E)$ over $E$ ( = "polynomials" on $E^{\prime}$ ), then a solution of the $m$-problem for $\phi$ will not define a measure on $E^{\prime}$ but merely a measure on the algebraic dual space $E^{*}$ of $E$ [22].

One sees that, in order to develop a step further our non-commutative generalization of the moment problem, we have to generalize the part of that problem corresponding to infinite dimensional measures on topological vector spaces. This will be done in a forthcoming paper.

Acknowledgements. The author is indebted to H. J. Borchers, H. Epstein, and J. Yngvason for numerous stimulating discussions and suggestions. It is a pleasure to thank them for their kind interest. We also thank H. Araki and A.Jaffe for their kind help in writing this revised manuscript.

Note. Soon after the publication of the Part I of this work [1], we realised that the condition of quasianalicity of the vacuum discussed there had already been introduced by Gachok, V.P.: Nuovo Cimento 45A, 158 (1966).

\section{References}

1. Dubois-Violette, M. : Commun. math. Phys. 43, 225-254 (1975)

2. Powers, R. T.: Commun. math. Phys. 21, 85-124 (1971)

3. Nussbaum, A.E.: Ark. Mat. 6, 179 (1965)

4. Akhiezer,N.I.: The classical moment problem. Edinburgh-London: Oliver and Boyd Ltd. 1965

5. Shohat,J.A., Tamarkin,J.D.: The problem of moments. Providence: American Mathematical Society 1963

6. Jaffe, A.M. : Dynamics of a cut-off $\lambda \phi^{4}$ field theory. Princeton: Thesis 1975

7. Ruelle,D.: Commun. math. Phys. 3, 133 (1966)

8. Landford III,O.E.: In Les Houches 1970. Statistical mechanics and quantum field theory (eds. C.de Witt, R.Stora). New York: Gordon and Breach 1971

9. Dixmier,J.: Les $C^{*}$-algèbres et leurs représentations. Paris: Gauthier-Villars 1964

10. Schaefer,H.H.: Topological vector spaces. Berlin-Heidelberg-New York: Springer 1971

11. Schwartz,L.: Théorie des distributions. Paris: Hermann 1966

12. Faris,W.G.: Self-adjoint operators. Berlin-Heidelberg-New York : Springer 1975

13. Araki,H.: Zürich lectures 1961-1962 (unpublished)

14. Wightman, A.S.: Ann. Inst. Henri Poincaré, Vol. I, 403 (1964)

15. Glimm,J., Jaffe, A. : Ann. Math. 91, 362 (1970)

16. Glimm,J., Jaffe, A.: J. Math. Phys. 13, 1568 (1972)

17. McBryan,O. A.: Nuovo Cimento 18, 654 (1973)

18. Glimm,J. Jaffe,A.: In Les Houches 1970. Statistical mechanics and quantum field theory (eds. C.de Witt, R.Stora). New York: Gordon and Breach 1971 
19. Haag, R., Kastler,D.: J. Math. Phys. 5, 848 (1964)

20. Jost, R.: The general theory of quantized fields. Providence American Mathematical Society 1965

21. Streater, R.F., Wightman,A.S.: PCT, spin and statistics, and all that. New York-Amsterdam: W. A. Benjamin Inc. 1964

22. Reed, M.C.: In "Ettore Majorana" 1973. Constructive quantum field theory (eds. G.Velo, A. Wightman). Berlin-Heidelberg-New York: Springer 1973

Communicated by H. Araki

Received July 28, 1975; in revised form December 27, 1976 